\title{
Uma Introdução aos Métodos de Cálculo da Energia de Casimir
}

\author{
J.J. Passos Sobrinho* e A.C. Tort ${ }^{\dagger}$ \\ Instituto de Física \\ Universidade Federal do Rio de Janeiro \\ Cidade Universitária, Ilha do Fundão, Caixa Postal 68528 \\ 21945-970 Rio de Janeiro, Brasil
}

Recebido em 20 de Março de 2001. Aceito em 09 de Outubro de 2001.

\begin{abstract}
O efeito Casimir é um dos aspectos mais intrigantes da física moderna. A previsão da existência de uma força macroscópica de origem quântica entre condutores neutros e sua posterior comprovação experimental é sem dúvida um dos triunfos da teoria quântica dos campos. Complementando uma introdução conceitual publicada recentemente nesta revista, apresentamos alguns métodos de cálculo da energia de Casimir, que é a grandeza fundamental que origina o efeito Casimir.
\end{abstract}

The Casimir effect is one of the most intriguing aspects of modern physics. The prediction of the existence of a macroscopic force of quantum origin between two neutral conductors and its subsequent experimental verification is without doubt the hallmark of quantum field theory. The present work complements a conceptual introduction recently published in this journal by presenting some of the methods of evaluation of the Casimir energy which gives rise to the Casimir effect.

\section{Introdução}

Em 1948 H.B.G. Casimir [1] previu que duas placas condutoras planas, paralelas e bem próximas sofreriam uma força de atração mesmo quando postas completamente descarregadas no vácuo. Tal força, que chamamos de força de Casimir, seria dada por:

$$
F=-A \frac{\pi^{2} \hbar c}{240 a^{4}}
$$

onde $A$ é a área de cada uma das placas, $a$ a separação entre elas e o sinal menos é uma indicação de que a força é atrativa. Para placas de $1 \mathrm{~cm}^{2}$ de área e separação de $1 \mu \mathrm{m}$, a força atrativa é de 0,013 dynas, muito pequena, porém observável. De fato, em $1958 \mathrm{M}$. Sparnaay [2] mediu tal força, embora seu experimento fosse muito pouco preciso. Contudo, em experimentos recentes realizados por Lamoreaux [3] e, de modo independente, por Mohideen e Roy [4], a força de Casimir foi medida com grande precisão. Essas medidas não deixam qualquer dúvida sobre a existência concreta do efeito descoberto por Casimir e que leva seu nome.

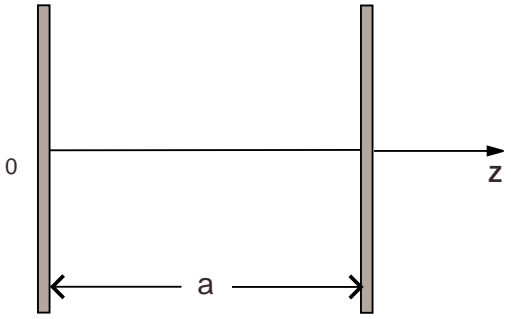

Figura 1. A introdução de placas condutoras neutras no vácuo quântico eletromagnético distorce as oscilações de ponto zero deste último. A conseqüência é uma força de atração macroscópica entre as placas, sendo este o efeito Casimir.

De acordo com Casimir [1], o vácuo quântico eletromagnético é alterado pela presença das placas metálicas. Essas impõem condições de contorno ao campo eletromagnético, mesmo ao que existe apenas como flutuações do vácuo quântico. As condições de contorno alteram a energia do vácuo e a alteração, que é chamada de energia de Casimir, é que dá origem ao efeito de atração entre as placas. A alteração de energia calculada por Casimir é dada por:

$$
E_{0}=-A \frac{\hbar c \pi^{2}}{720 a^{3}}
$$

*jandir@if.ufrj.br

†tort@if.ufrj.br 
Derivando-se essa energia em relação à separação $a$, obtém-se a força atrativa dada por (1).

Embora o efeito Casimir original seja um efeito do campo eletromagnético, todo campo relativístico quântico pode sofrer uma alteração de energia do seu vácuo quando submetido a condições de contorno. Tal alteração também é chamada de energia de Casimir, assim como o efeito dela decorrente é chamado de efeito Casimir do campo em questão. Neste trabalho, consideraremos o efeito Casimir de um campo escalar. O objetivo será fazer uma apresentação didática de alguns dos métodos de cálculo da energia de Casimir. O presente trabalho deve ser visto como uma continuação de um trabalho mais conceitual publicado nesta revista [26].

Dissemos que a energia de Casimir é a alteração da energia do vácuo de um campo quântico quando este é submetido a condições de contorno. Se o espectro de energias do sistema é dado pelas freqüências $\omega_{n}$, onde $n$ varre um determinado conjunto de índices, teremos que a energia de Casimir será dada por:

$E_{\text {Casimir }}=\left[\sum_{n} \frac{\hbar \omega_{n}}{2}\right]_{\text {com c. de c. }}-\left[\sum_{n} \frac{\hbar \omega_{n}}{2}\right]_{\text {sem c. de c. }}$,

onde com c. de c. indica que na primeira soma, o espectro é o do sistema submetido a condições de contorno, enquanto que sem c. de c. indica que, na segunda soma, o espectro é o do sistema livre de condições de contorno. Entretanto, essa definição de energia de Casimir, e outras equivalentes, é problemática, pois cada uma das somas acima é divergente assim como a diferença entre elas. Trata-se de quantidades mal definidas. Para dar sentido físico a essas quantidades, é necessário realizar nelas o que costuma ser chamado de renormalização. A renormalização de uma quantidade é feita normalmente em três etapas. A primeira é chamada de regularização da quantidade mal definida. Para regularizar uma quantidade $Q$ mal definida, devemos definir uma nova quantidade dependente de um novo parâmetro; digamos que $\lambda$ seja o novo parâmetro e $Q(\lambda)$ a nova quantidade. Dizemos que $Q(\lambda)$ é a regularização de $Q$ se possuir as seguintes propriedades: (i) $Q(\lambda)$ tem $Q$ por limite quando $\lambda$ tende para algum limite $\lambda_{0}$ e (ii) $Q(\lambda)$ é bem definida quando $\lambda \neq \lambda_{0}$. O parâmetro $\lambda$ é chamado neste contexto de parâmetro de regularização. A segunda etapa no processo de renormalização consiste em subtrair da expressão regularizada uma quantidade $Q_{e s p}(\lambda)$, que é bem definida devido à regularização e que a teoria nos indica como espúria, obtendo $Q(\lambda)-Q_{e s p}(\lambda)$. A terceira etapa consiste em tomar o limite dessa diferença quando $\lambda \rightarrow \lambda_{0}$. Esse limite é o que chamamos de valor renormalizado da grandeza $Q$, originalmente mal definida. O valor renormalizado é aquele ao qual atribuímos significado físico e supomos em tese, mensurável. A energia de Casimir fornece um exemplo simples do processo de renormalização. Como é fácil notar, ela é a renormalização da energia do vácuo sob condições de contorno. De fato, o primeiro somatório em (3) é essa energia, em geral, mal definida. Seja

$$
\left[\sum_{n} \frac{\hbar \omega_{n}}{2}\right]_{\text {com } c . d e c}^{(\lambda)}
$$

sua versão regularizada pelo parâmetro de regularização $\lambda$. No nosso contexto de teoria de campos, a energia do vácuo puro e simples, sem condições de contorno ou outra influências externas é uma grandeza espúria. Ela é o segundo somatório em (3), que representamos por

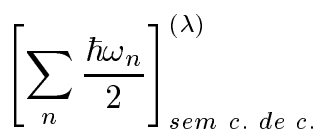

sua expressão regularizada. Ao contrário de (3), a diferença:

$$
\left[\sum_{n} \frac{\hbar \omega_{n}}{2}\right]_{\text {com c. de c. }}^{(\lambda)}-\left[\sum_{n} \frac{\hbar \omega_{n}}{2}\right]_{\text {sem c. de c. }}^{(\lambda)},
$$

é, por definição de regularização, livre de infinidades e perfeitamente regular, embora não tenha sentido físico, pois depende da regularização utilizada e, portanto, em última análise, de quem está fazendo o cálculo e escolheu a regularização em questão. Tomando, então, o limite em que desaparece o parâmetro da regularização obtemos a energia renormalizada do vácuo sob condições de contorno, que é a energia de Casimir:

$$
E_{\text {Casimir }}^{(\text {ren. })}=\lim _{\lambda \rightarrow \lambda_{0}}\left\{\left[\sum_{n} \frac{\hbar \omega_{n}}{2}\right]_{\text {com c. de c. }}^{(\lambda)}-\left[\sum_{n} \frac{\hbar \omega_{n}}{2}\right]_{\text {sem c. de c. }}^{(\lambda)}\right\}
$$


O processo de renormalização depende, é claro, da regularização utilizada. A regularização pode ser feita de várias maneiras, que são conhecidas na literatura especializada como métodos ou técnicas de regularização. São os métodos de regularização que permitem atribuir um siginificado físico sensato à (3), e às definições que lhe são equivalentes. Outras quantidades divergentes que aparecem quando aplicamos a teoria quântica de campos em outros contextos também ganham sentido físico graças aos métodos de regularização. Como conseqüência dos esforços de várias gerações de físicos teóricos, hoje em dia temos ao nosso dispor um certo número de métodos de regularização que podem ser empregados, de acordo com o problema em questão, com maior ou menor eficácia. Acreditamos firmemente que os resultados fisicamente relevantes não dependem dos métodos particulares de regularização e renormalização que foram escolhidos.

Neste trabalho, queremos introduzir o leitor a quatro métodos de regularização que permitem cálcular energias de Casimir, a saber:

(a) o método do corte nas freqüências;

(b) o método da discretização do espaço (ou regularização na rede);

(c) o método da função zeta generalizada;

(d) e o método da função de Green.
Exemplificaremos cada um desses métodos com sistemas simples: o ubíquo oscilador harmônico simples e o campo escalar real sem auto-interação ou outras interações hipotéticas, exceto as que estão sendo simuladas pelas condições de contorno que lhe serão impostas. Essa escolha de exemplos permitirá que ressaltemos os aspectos principais das etapas assinaladas acima, sem que a nossa atenção seja desviada por detalhes que não são importantes à compreensão dos métodos. A lista acima não esgota os métodos de cálculo, pois a ela poderíamos acrescentar, por exemplo, o método da regularização dimensional ou os métodos locais, como o da função zeta local, mas a sua inclusão e sua discussão detalhada tornaria este trabalho demasiadamente longo.

Como é costumeiro em teoria quântica de campos, faremos uso de unidades naturais, mantendo a constante de Planck $\hbar$ e a velocidade da luz $c$ iguais à unidade em todos os cálculos. Em unidades naturais a energia tem dimensão do inverso do comprimento. Para restaurar a unidade de energia usual, basta multiplicar o resultado dado em unidades naturais pelo fator $\hbar c$.

\section{A energia de Casimir}

Comecemos por recordar como a energia do vácuo surge na teoria quântica de campos. Consideremos, para fixar idéias, um campo escalar real e livre em $3+1$ dimensões. A densidade lagrangiana desse campo é dada por:

$$
\mathcal{L}=\frac{1}{2}\left(\frac{\partial \varphi(\mathbf{x}, t)}{\partial t}\right)^{2}-\frac{1}{2} \boldsymbol{\nabla} \varphi(\mathbf{x}, t) \cdot \nabla \varphi(\mathbf{x}, t)-\frac{1}{2} m^{2} \varphi^{2}(\mathbf{x}, t),
$$

onde $m$ é a massa da partícula escalar associada ao campo. Das equações de Euler-Lagrange segue-se, então, a equação de movimento clássica para o campo $\varphi(\mathbf{x}, t)$, que se lê:

$$
\frac{\partial^{2} \varphi(\mathbf{x}, t)}{\partial t^{2}}-\nabla^{2} \varphi(\mathbf{x}, t)+m^{2} \varphi(\mathbf{x}, t)=0 .
$$

Na ausência de condições de contorno, escrevemos a solução geral dessa equação como uma combinação linear de ondas planas:

$$
\varphi(\mathbf{x}, t)=\int \frac{d^{3} \mathbf{k}}{(2 \pi)^{3} \sqrt{2 \omega_{\mathbf{k}}}}\left[a_{\mathbf{k}} \exp \left(i \mathbf{k} \cdot \mathbf{x}-i \omega_{\mathbf{k}} t\right)+a_{\mathbf{k}}^{*} \exp \left(-i \mathbf{k} \cdot \mathbf{x}+i \omega_{\mathbf{k}} t\right)\right],
$$

onde $\mathbf{k}$ é o vetor de onda, $\omega_{\mathbf{k}}=\sqrt{\mathbf{k}^{2}+m^{2}}$, de acordo com a relação de dispersão do campo livre; $a_{\mathbf{k}}$ e $a_{\mathbf{k}}^{*}$ são as amplitudes das ondas planas, e $a_{\mathbf{k}}^{*}$, é o complexo conjugado de $a_{\mathbf{k}}$ para garantir o caráter real do campo escalar. $\mathrm{O}$ momento canônico por unidade de volume é dado por $\pi=\partial \mathcal{L} / \partial \varphi / \partial t$, e obtemos por meio de (10):

$$
\pi(\mathbf{x}, t)=(-i) \int \frac{d^{3} \mathbf{k}}{(2 \pi)^{3}} \sqrt{\frac{\omega_{\mathbf{k}}}{2}}\left[a_{\mathbf{k}} \exp \left(i \mathbf{k} \cdot \mathbf{x}-i \omega_{\mathbf{k}} t\right)-a_{k}^{*} \exp \left(-i \mathbf{k} \cdot \mathbf{x}+i \omega_{\mathbf{k}} t\right)\right] .
$$


A quantizacão do campo escalar segue o procedimento canônico, que consiste em transformar o campo e seu momento conjugado em operadores denotados por $\hat{\varphi}(\mathbf{x}, t)$ e $\hat{\pi}(\mathbf{x}, t)$, respectivamente, e postular para eles as relações de Heisenberg para tempos iguais:

$$
\begin{gathered}
{\left[\hat{\varphi}(\mathbf{x}, t), \hat{\pi}\left(\mathbf{x}^{\prime}, t\right)\right]=i \delta\left(\mathbf{x}-\mathbf{x}^{\prime}\right),} \\
{\left[\hat{\varphi}(\mathbf{x}, t), \hat{\varphi}\left(\mathbf{x}^{\prime}, t\right)\right]=\left[\hat{\pi}(\mathbf{x}, t), \hat{\pi}\left(\mathbf{x}^{\prime}, t\right)\right]=0 .}
\end{gathered}
$$

A quantização canônica do campo escalar implica na transformação em operadores dos coeficientes de Fourier em (10) e (11). O coeficiente $a_{\mathbf{k}}$ torna-se o operador $\hat{a}_{\mathbf{k}}$ e o coeficiente $a_{\mathbf{k}}^{*}$ torna-se o operador $\hat{a}_{\mathbf{k}}^{\dagger}$, que é o hermitiano conjugado de $\hat{a}_{\mathbf{k}}$. Em termos desses operadores, também chamados de osciladores do campo, as relações de Heisenberg (12) e (13) tomam a forma:

$$
\begin{gathered}
{\left[\hat{a}_{\mathbf{k}}, \hat{a}_{\mathbf{k}^{\prime}}^{\dagger}\right]=(2 \pi)^{3} \delta\left(\mathbf{k}-\mathbf{k}^{\prime}\right),} \\
{\left[\hat{a}_{\mathbf{k}}, \hat{a}_{\mathbf{k}^{\prime}}\right]=\left[\hat{a}_{\mathbf{k}}^{\dagger}, \hat{a}_{\mathbf{k}^{\prime}}^{\dagger}\right]=0 .}
\end{gathered}
$$

O hamiltoniano da teoria é obtido do lagrangeano (8) pelo procedimento usual de efetuar uma transformação de Legendre. Depois de quantizado, o hamiltoniano assume a forma:

$$
\hat{H}=\frac{1}{2} \int d^{3} \mathbf{x}\left[\hat{\pi}^{2}+(\nabla \hat{\varphi})^{2}+m^{2} \hat{\varphi}^{2}\right] .
$$

Fazendo-se uso da versão quantizada de (10) e (11), esse hamiltoniano pode ser expresso em termos dos operadores $\hat{a}_{\mathbf{k}}(t)$ e $\hat{a}_{\mathbf{k}}^{\dagger}$. O resultado é:

$$
\hat{H}=\int d^{3} \mathbf{k}\left(\hat{a}_{\mathbf{k}}^{\dagger} \hat{a}_{\mathbf{k}}+\frac{1}{2}\right) \omega_{\mathbf{k}} .
$$

Como podemos inferir da forma da (17), a quantização canônica de um campo escalar real livre mostra-nos que ele pode ser considerado como uma coleção formada por um número infinito de osciladores harmônicos quantizados independentes, uma vez que a hamiltoniana do campo é uma soma de hamiltonianas de osciladores harmônicos de diferentes freqüências. Os operadores $\hat{a}_{\mathbf{k}}(t)$ e $\hat{a}_{\mathbf{k}}^{\dagger}(t)$, assim como $\hat{\varphi}(\mathbf{x}, t)$ e $\hat{\pi}(\mathbf{x}, t)$, atuam sobre vetores que pertencem a um particular espaço vetorial complexo chamado espaço de Fock, ou de número de ocupação. Este espaço vetorial é um produto direto dos espaços de Hilbert associados a cada oscilador independente que representa um modo normal de vibração do campo.

A imposição de condições de contorno no campo escalar afeta sua parte espacial e, portanto, modifica os possíveis modos normais de vibração do campo e suas respectivas freqüências. Os modos normais são então dados por certas funções modais $\mathbf{F}_{\mathbf{k}}(\mathbf{x})$ e freqüências $\omega_{\mathbf{k}}$, onde agora $\mathbf{k}$ assume apenas os valores ditados pelas condições de contorno. O campo assume então a forma:

$$
\hat{\phi}(\mathbf{x}, t)=\sum_{\mathbf{k}}\left[\hat{a}_{\mathbf{k}} F_{\mathbf{k}}(\mathbf{x}) \exp \left(-i \omega_{\mathbf{k}} t\right)+\hat{a}_{\mathbf{k}}^{\dagger} F_{\mathbf{k}}^{*}(\mathbf{x}) \exp \left(i \omega_{\mathbf{k}} t\right)\right]
$$

que devemos comparar com (10). Notemos que, em (18), usamos o símbolo de somatório - que também usaremos na (21) - enquanto que, em (10), foi usado o símbolo de integração sobre k; com isto queremos indicar que condições de contorno em geral discretizam o espectro. As funções modais $F_{\mathbf{k}}(\mathbf{x})$ substituem as exponenciais harmônicas, que são as funções modais na ausência de condições de contorno. O somatório estende-se sobre os valores de $\mathbf{k}$ ditados pelas condições de contorno. As funções modais $F_{\mathbf{k}}(\mathbf{x})$ são determinadas resolvendo-se a equação de Helmholtz associada com a (9), acrescida das ditas condições de contorno:

$$
\nabla^{2} F_{\mathbf{k}}(\mathbf{x})+\mathbf{k}^{2} F_{\mathbf{k}}(\mathbf{x})=0
$$

As funções modais formam um conjunto completo e ortonormal:

$$
\int d^{3} \mathbf{x} F_{\mathbf{k}}^{*}(\mathbf{x}) F_{\mathbf{k}^{\prime}}(\mathbf{x})=\delta_{\mathbf{k} \mathbf{k}^{\prime}}
$$

As modificações no campo ocasionadas pelas condições de contorno nos levam agora à expressão análoga a (17), dada por:

$$
\hat{H}=\sum_{\mathbf{k}}\left(\hat{a}_{\mathbf{k}}^{\dagger} \hat{a}_{\mathbf{k}}+\frac{1}{2}\right) \omega_{\mathbf{k}} .
$$

onde a soma estende-se aos valores de $\mathbf{k}$ ditados pelas condições de contorno. No estado de vácuo, nenhum modo normal está excitado e a energia é dada apenas pelo termo $\omega_{\mathbf{k}} / 2$ em (16) e (21), nos casos em que, respectivamente, há ou não há condições de contorno. Desse modo obtemos a energia de Casimir (7). Passemos, então, a alguns métodos de regularização e renormalização usados para calcular essa energia. 


\section{O método do corte}

Consideremos, por simplicidade, um campo escalar real $\hat{\varphi}(x, t)$, sem massa, em uma dimensão temporal e uma dimensão espacial, isto é, em $1+1$ dimensões espaçotemporais. A solução da equação Helmholtz unidimensional pode ser escrita na forma:

$$
\begin{aligned}
F_{k}(x) & =C \exp (i k x)+C^{*} \exp (-i k x) \\
& =A \cos (k x)+B \sin (k x)
\end{aligned}
$$

Vamos supor que as condições de contorno impostas à parte espacial do campo escalar sejam as de Dirichlet em $x=0$ e em $x=a$. Nesse caso, segue que $F_{k}(0)=F_{k}(a)=0$. A solução dada pela (22) reduz-se então a:

$$
F_{n}(x)=B \sin \left(\frac{n \pi x}{a}\right),
$$

onde $n \in \mathbb{N}=\{1,2,3, \ldots\}$ e as freqüências normais são dadas por $\omega_{n}=\left|k_{n}\right|=n \pi / a$. A energia do vácuo a ser regularizada é:

$$
E_{0}(a)=\frac{1}{2} \sum_{n \in \mathbb{N}} \frac{n \pi}{a}
$$

A regularização por corte consiste na introdução de um fator no somatório que torne a soma convergente. Tal fator corta a contribuição de parte do espectro das freqüências, daí o nome desse método de regularização. Escolheremos, aqui, o fator como sendo $\exp \left(-\lambda \omega_{n}\right)$, onde $\omega_{n}=n \pi / a$ e $\lambda$ é um número real positivo. Observemos que o corte exponencial é uma das possibilidades, talvez a mais simples, mas não a mais geral. Usando-o na equação anterior, podemos escrever:

$$
\begin{aligned}
E_{0}(a, \lambda) & =\frac{1}{2} \sum_{n \in \mathbb{N}} \frac{n \pi}{a} \exp (-\lambda n \pi / a) \\
& =-\frac{1}{2} \frac{d}{d \lambda} \sum_{n \in \mathbb{N}} \exp (-\lambda n \pi / a)
\end{aligned}
$$

O somatório pode ser identificado como uma progressão geométrica formada por um número infinito de termos cuja razão é $\exp (-\lambda \pi / a)$ e sua fórmula de soma:

$$
\sum_{k \in \mathbb{N}} q^{k}=\frac{q}{1-q}, \quad|q|<1 .
$$

Somando tal progressão, obtemos:

$$
E_{0}(a, \lambda)=-\frac{1}{2} \frac{d}{d \lambda}\left[\frac{\exp (-\lambda \pi / a)}{1-\exp (-\lambda \pi / a)}\right]
$$

É conveniente que, antes de efetuar a derivação indicada, exploremos um pouco mais o termo entre colchetes na (27). Os polinômios de Bernoulli $B_{k}(x)$ são definidos por [5]:

$$
\frac{t \exp (x t)}{\exp (t)-1}=\sum_{k \in \mathbb{N}-1} B_{k}(x) \frac{t^{k}}{k !}
$$

Usando essa fórmula em (27), obtemos:

$$
\begin{aligned}
E_{0}(a, \lambda) & =\frac{1}{2} \frac{d}{d \lambda} \sum_{k \in \mathbb{N}-1} \frac{B_{k}(1)}{k !}\left(-\frac{\lambda \pi}{a}\right)^{k-1} \\
& =\frac{1}{2} \frac{d}{d \lambda}\left(-\frac{B_{0}}{0 !} \frac{a}{\lambda \pi}+\frac{B_{1}}{1 !}-\frac{B_{2}}{2 !} \frac{\lambda \pi}{a}\right)+O\left(\lambda^{3}\right)
\end{aligned}
$$

onde usamos a abreviação $B_{k}=B_{k}(1)$. Temos que $B_{0}=1, B_{1}=-1 / 2$ e $B_{2}=1 / 6$ [5]. Fazendo a derivada em (29), obtemos:

$$
E_{0}(\lambda, a)=\frac{1}{2 \pi} \frac{a}{\lambda^{2}}-\frac{\pi}{24} \frac{1}{a}+O\left(\lambda^{2}\right) .
$$

A seguir, redefiniremos (renormalizaremos) a energia do vácuo no espírito da definição dada por (7). Considere- mos dois universos unidimensionais finitos, um formado por uma cavidade unidimensional de comprimento $L$ com uma partição que a subdivide em uma cavidade de comprimento $a$, outra de comprimento $L-a$, (veja a Fig. 2) e outro universo formado por uma segunda cavidade de comprimento $L$. A definição dada por (3) pode ser rescrita na forma:

$$
E_{0}(a)=\lim _{\lambda \rightarrow 0, L \rightarrow \infty}\left[E_{0}(a, \lambda)+E_{0}(L-a, \lambda)-E_{0}(L, \lambda)\right]
$$


onde:

$$
E_{0}(L-a, \lambda)=\frac{1}{2 \pi} \frac{(L-a)}{\lambda^{2}}-\frac{\pi}{24} \frac{1}{(L-a)}+O\left(\lambda^{2}\right),
$$

e:

$$
E_{0}(L, \lambda)=\frac{1}{2 \pi} \frac{L}{\lambda^{2}}-\frac{\pi}{24} \frac{1}{L}+O\left(\lambda^{2}\right),
$$

Fazendo uso da (31), obtemos o resultado físico procurado:

$$
E_{0}(a)=-\frac{\pi}{24 a} \text {. }
$$

Conseqüentemente, a força de Casimir é:

$$
F(a)=-\frac{\partial E_{0}(a)}{\partial a}=-\frac{\pi}{24 a^{2}} .
$$

O sinal algébrico negativo indica que a força entre os pontos da fronteira é atrativa. O resultado final dado por (34) poderia ser parcialmente antecipado por meio da utilização de argumentos dimensionais, os quais nos teriam levado a concluir que a energia do vácuo deveria ser proporcional a $1 / a$, já que em unidades naturais a energia tem dimensões do inverso do comprimento. Entretanto, o sinal algébrico e o fator numérico corretos só podem ser obtidos por meio de cálculos explícitos. Esta impossibilidade de antecipar o caráter atrativo ou repulsivo da força de Casimir, que depende do tipo de campo estudado e das condições de contorno impostas a esse campo, é conhecida como o "mistério da força de Casimir" [7].

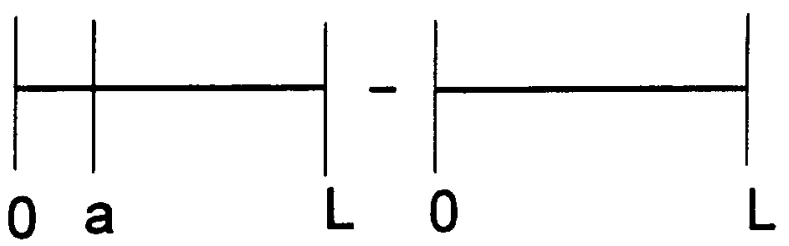

Figura 2. Esquema de subtração para a obtenção da energia de Casimir de um campo escalar sem massa em $1+1$ dimensões e que obedece condições de Dirichlet em $x=0, a$ e $L$.

\section{O método da regularização na rede}

Uma vez que a origem da divergência da energia do vácuo está na soma da energia de ponto zero de um número infinito de osciladores obtidos na quantização do campo em questão, uma abordagem possível consiste na redução deste número infinito de graus de liberdade a um número finito. Isso pode ser feito considerandose o espaço, não contínuo, mas sim discreto, i.e.: como se este fosse uma rede tridimensional formada por pontos que mantêm uma distância fixa entre si. Por esta razão, esse método é chamado de regularização na rede. Em uma dimensão espacial, o processo de discretização do espaço leva-nos a substituir a equação de movimento clássica para o campo em questão por um sistema de $N$ equações diferenciais ordinárias acopladas, com $N \in \mathbb{N}$. Na interpretação usual, cada uma dessas equações representa um oscilador acoplado a seus vizinhos adjacentes. Em seguida, diagonalizamos o sistema reduzindo-o a um conjunto de osciladores harmônicos desacoplados que são então quantizados. A energia do ponto zero é a soma das freqüências normais multiplicada pelo fator $1 / 2$. Convém ressaltar que esse método é aplicado no contexto da mecânica clássica no estudo dos modos normais de um sistema constituído por massas discretas e idênticas acopladas [6].

Como exemplo concreto, consideremos o campo escalar real $\varphi$ em $1+1$ dimensões da seção anterior. A equação de movimento clássica para tal campo é a equação da onda:

$$
\frac{\partial^{2} \varphi(x, t)}{\partial x^{2}}-\frac{\partial^{2} \varphi(x, t)}{\partial t^{2}}=0 .
$$

Suponhamos que condições de contorno sejam impostas sobre o campo em $x=0$ e $x=L$. Essas condições podem ser condições de Dirichlet, Neuman, mistas, periódicas ou mesmo antiperiódicas. Deixemo-las, no momento, em aberto. Essencialmente, o método consiste em substituir os valores do campo no intervalo $[0, L]$ por um conjunto de $N+2$ valores em $N+2$ pontos intervalos $(N \in \mathbb{N})$. Os pontos são igualmente espaçados entre si por uma distância $d>0$, denominada de parâmetro de rede. Nesse espaço unidimensional discreto, cada ponto recebe um endereço dado por $x_{j}=j d$, onde $j \in\{0,1,2, \ldots, N+1\}$. O campo, com todos os seus valores no intervalo, é recuperado fazendo-se $N \rightarrow \infty$ e $d \rightarrow 0$, mas de tal forma que a quantidade $(N+1) d=L$ permaneça constante. Esse limite é tomado ao final dos cálculos para obter a energia de Casimir do campo.

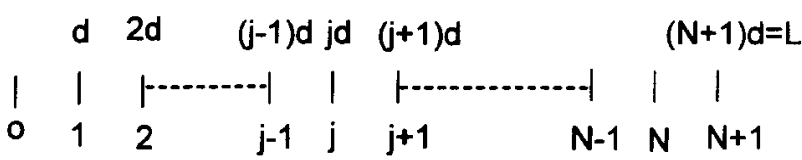

Figura 3. A discretização do espaço. Em uma dimensão espacial, o número de freqüências normais é finito e igual ao número de graus de liberdade $N$.

Para discretizar a equação da onda, fazemos as substituições: $x \mapsto x_{j}, \varphi(x, t) \mapsto \varphi\left(x_{j}, t\right) \equiv \varphi_{j}(t) \mathrm{e:}$

$$
\frac{\partial \varphi(x, t)}{\partial x} \mapsto \frac{\varphi_{j}(t)-\varphi_{j-1}(t)}{d}
$$


e ainda,

$$
\frac{\partial^{2} \varphi\left(x_{j}, t\right)}{\partial x^{2}} \mapsto \frac{1}{d}\left[\frac{\varphi_{j+1}(t)-\varphi_{j}(t)}{d}-\frac{\varphi_{j}(t)-\varphi_{j-1}(t)}{d}\right]
$$

Nesse caso, a equação de onda para o campo escalar discretizada transforma-se em um sistema de equações diferenciais ordinárias acopladas que se lê:

$$
\frac{d^{2} \varphi_{j}(t)}{d t^{2}}+2 \omega_{0}^{2} \varphi_{j}(t)-\omega_{0}^{2}\left(\varphi_{j+1}(t)+\varphi_{j-1}(t)\right)=0
$$

onde definimos $\omega_{0}^{2}:=1 / d^{2}$. As equações acima são totalmente análogas às equações de movimento para uma rede unidimensional formada por partículas de massas idênticas unidas por molas idênticas, ou, se for o caso, contas idênticas presas a uma corda tensa de massa nula. Adotando o procedimento usual de solução deste sistema [6], escrevemos a solução tentativa:

$$
\varphi_{j}(t)=C \exp i(\theta j \pm \omega t)
$$

com $C \in \mathbb{C}$ e $\theta$ e $\omega$ a serem determinados. Substituindo essa expressão para o campo em (39), obtemos:

$$
\omega=2 \omega_{0} \sin \frac{\theta}{2}
$$

Para determinar completamente a solução, devemos especificar as condições de contorno. Como um exemplo, tomemos novamente as condições de Dirichlet. Traduzidas em termos de condições de contorno sobre o campo na rede, temos: $\varphi_{0}(t)=0$, e $\varphi_{N+1}(t)=0$. Para aplicálas, é conveniente escrever (40) na forma:

$$
\varphi_{j}(t)=[D \cos (j \theta)+E \sin (j \theta)] \exp ( \pm i \omega t),
$$

Segue, então, que:

$$
\phi_{0}(t)=D \exp ( \pm i \omega t)=0,
$$

que leva a $D=0 \mathrm{e}$,

$$
E \sin [(N+1) \theta] \exp ( \pm i \omega t)=0 .
$$

Conseqüentemente, temos que $\theta$ é dado por:

$$
\theta_{n}=\frac{n \pi}{(N+1)}, \quad(n=1,2, \ldots N),
$$

já que inteiros não-positivos geram soluções linearmente dependentes e valores de $n$ tais que $n=N+1, N+2, \ldots$ também não contribuem pelo mesmo motivo. Este fato não deve ser surpresa, pois em uma dimensão, como não há degenerescência, os $N$ graus de liberdade correspondem exatamente a $N$ modos de vibração. A solução dada por (42) então será:
$\phi_{j}^{(n)}(t)=A^{(n)} \sin \left(j \theta_{n}\right) \exp \left( \pm i \omega_{n} t\right), \quad(j=1,2, \ldots N)$,

onde,

$$
\omega_{n}=2 \omega_{0} \sin \left(\frac{\theta_{n}}{2}\right), \quad(n=1,2, \ldots N)
$$

são as freqüências dos modos normais $\operatorname{com} \theta_{n}$ dado por (45). A energia de ponto zero é dada pela soma dessas frequiências:

$$
\begin{aligned}
E_{0} & =\omega_{0} \sum_{n=1}^{N} \sin \left(\frac{\theta_{n}}{2}\right) \\
& =\omega_{0} \Im \sum_{n=1}^{N} \exp \left(i \frac{\theta_{n}}{2}\right) .
\end{aligned}
$$

Agora, utilizando a fórmula de soma de uma progressão geométrica com um número de termos finito:

$$
\sum_{k=1}^{N} q^{k-1}=\frac{\left(q^{N}-1\right)}{q-1}, \quad q \neq 1,
$$

e definindo o ângulo $\delta$ pela relação:

$$
n \delta: \equiv \frac{\theta_{n}}{2}=\frac{n \pi}{2(N+1)},
$$

obtemos, após algumas manipulações:

$$
\begin{aligned}
E_{0} & =\omega_{0} \Im \exp (i \delta) \frac{[\exp (i \delta N)-1]}{\exp (i \delta)-1} \\
& =\omega_{0} \sin \left[(N+1) \frac{\delta}{2}\right] \frac{\sin \left(\frac{N \delta}{2}\right)}{\sin \left(\frac{\delta}{2}\right)} . \\
& =\frac{1}{2} \omega_{0}\left[\cot \left(\frac{\delta}{2}\right)-1\right] .
\end{aligned}
$$

Substituindo $\delta$ por sua definição, chegamos a:

$$
E_{0}(L)=\frac{1}{2} \omega_{0}\left[\cot \left(\frac{\pi d}{4 L}\right)-1\right] .
$$

Utilizando a expansão da cotangente próximo a $x=0$ [5]:

$$
\cot x=\frac{1}{x}-\frac{1}{3} x-\frac{1}{45} x^{3}+O\left(x^{5}\right)
$$

obtemos finalmente de (52) o resultado:

$$
\begin{aligned}
E_{0}(L) & =\frac{2 L}{\pi d^{2}}-\frac{\pi}{24 L}-\frac{\pi^{3} d^{2}}{5760 L^{3}}+\cdots \\
& \approx \frac{2 L}{\pi d^{2}}-\frac{\pi}{24 L},
\end{aligned}
$$

onde já desprezamos os termos que se anularão no limite contínuo da rede $(d \rightarrow 0$ e $N \rightarrow \infty)$.

Após esses resultados preliminares, passemos ao cáculo da energia de Casimir. Consideremos, por conveniência, uma rede unidimensional de comprimento $2 L$ 
com o objetivo de, no final, tomar o limite $L \rightarrow \infty$ para que o campo seja definido em todo o espaço. Suponhamos, ainda, que as condições de contorno de Dirichlet sejam impostas nos pontos $x=-L, 0, a$ e $x=L$. A definição de energia de Casimir associada a essa rede é, então, dada por:

$E_{0}=\lim _{L \rightarrow \infty}\left[E_{0}(|-L|)+E_{0}(a)+E_{0}(L-a)-E_{0}(2 L)\right]$,

veja a Fig. 4. Fazendo as substituições necessárias e tomando o limite contínuo, obtemos o resultado final:

$$
E_{0}(a)=-\frac{\pi}{24 a},
$$

que está em acordo com o obtido com o método do corte. Esse resultado é o obtido por Cooke [8] para o efeito Casimir em $1+1$

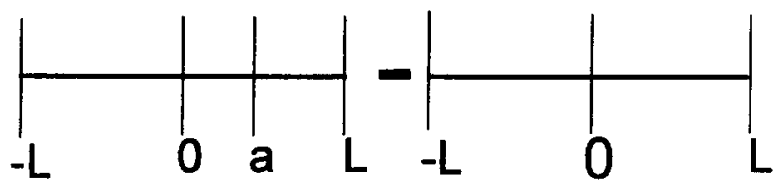

Figura 4. Esquema de subtração para a obtenção da energia de Casimir.

\section{A energia do vácuo como o lo- garitmo de um determinante}

Uma maneira elegante e eficaz de calcular a energia de Casimir consiste em expressá-la como o logaritmo de um determinante que é definido pela teoria em consideração, seja por meio de seu lagrangeano seja por meio de sua equação de movimento. Esta é geralmente dada por um operador do tipo dito elíptico e o determinante é dado diretamente em termos desse operador. Seguindo [13], começaremos recordando um pouco das formulações de Schrödinger e Heisenberg de um sistema com um grau de liberdade classicamente descrito pela coordenada cartesiana $x$ e pelo momento linear $p$. O lagrangeano clássico associado a tal sistema é dado por:

$$
L=\frac{m}{2}\left(\frac{d x}{d t}\right)^{2}-V(x) .
$$

onde $V(x)$ é o potencial ao qual a partícula de massa $m$ está submetida.

Do ponto de vista da mecânica quântica, o sistema é descrito pelos operadores hermitianos independentes do tempo $\hat{X}_{S}$ e $\hat{P}_{S}$ na formulação de Schrödinger, e pelos operadores hermitianos dependentes do tempo $\hat{X}_{H}(t)$ e $\hat{P}_{H}(t)$ na formulação de Heisenberg. Na verdade, o sistema admite uma infinidade de formulações, todas elas relacionadas entre si por meio de transformações unitárias. Consideremos, no momento, apenas os operadores de posição nas duas formulações mencionadas. $\mathrm{Na}$ formulação de Schrödinger, os autovetores $|x\rangle_{S}$ de $\hat{X}_{S}$ são independentes do tempo e satisfazem à equação de autovalores:

$$
\hat{X}_{S}|x\rangle_{S}=x|x\rangle_{S}
$$

onde $x$ é um número real que representa o autovalor de posição associado ao autovetor $|x\rangle_{S}$. O autovalor $x$ é um resultado possível de uma medida de posição sobre a reta. O mesmo problema na formulação de Heisenberg se escreve:

$$
\hat{X}_{H}(t)|x, t\rangle_{H}=x|x, t\rangle_{H},
$$

onde denotamos $|x, t\rangle_{H}$ o autovetor de $\hat{X}_{H}(t)$ associado ao autovalor $x$. As formulações de Schrödinger e Heisenberg conectam-se por meio da transformação unitária:

$$
\hat{U}(t)=\exp (i \hat{H} t),
$$

onde $\hat{H}=\hat{H}(t)$ é o operador hamiltoniano que descreve a dinâmica do sistema e é da forma:

$$
\hat{H}=\frac{\hat{P}_{S}^{2}}{2 m}+\hat{V}\left(\hat{X}_{S}\right)
$$

Os autovetores de posição associados ao mesmo autovalor $x$ nas duas formulações relacionam-se por:

$$
|x, t\rangle=\exp (i \hat{H} t)|x\rangle
$$

e os operadores de posição por:

$$
\hat{X}_{H}(t)=\exp (i \hat{H} t) \hat{X}_{S} \exp (-i \hat{H} t) .
$$

Suponhamos, agora, que no instante $t_{1}$ medimos a posição da partícula e obtemos o autovalor $x_{1}$. Qual é a amplitude de probabilidade de que no instante $t_{2}$ a encontremos na posição $x_{2}$ ? Essa amplitude pode ser escrita na formulação de Heisenberg ou de Schrödinger. De fato, de (62) temos:

$$
\left\langle x_{2}, t_{2} \mid x_{1}, t_{1}\right\rangle=\left\langle x_{2}\left|\exp \left[-i \hat{H}\left(t_{2}-t_{1}\right)\right]\right| x_{1}\right\rangle .
$$

Pode-se mostrar que, para sistemas quânticos descritos por hamiltonianos da forma dada por (61), o lado direito da equação acima pode ser escrito em termos da soma de todas as curvas no espaço-tempo $x: t \mapsto x(t)$, que unem os pontos 1 e 2, isto é, da soma de todos os movimentos clássicos imagináveis, reais ou não, que começam em $x_{1}$ no instante $t_{1}$ e terminam em $x_{2}$ no instante $t_{2}$, veja a Fig. 5. Cada curva tem peso unitário e uma fase que depende do funcional ação avaliado sobre a curva em questão. Formalmente, a resposta à pergunta formulada acima se escreve [14]: 


$$
\begin{aligned}
\left\langle x_{2}, t_{2} \mid x_{1}, t_{1}\right\rangle & =\int_{x\left(t_{1}\right)=x_{1}}^{x\left(t_{2}\right)=x_{2}} D[x] \exp (i S[x]) \\
& =\int_{x\left(t_{1}\right)=x_{1}}^{x\left(t_{2}\right)=x_{2}} D[x] \exp \left(i \int_{t_{1}}^{t_{2}} d t L[x(t), \dot{x}(t)]\right),
\end{aligned}
$$

onde o símbolo $D[x]$ denota a medida usada nesta integral peculiar.

Elaboremos um pouco mais a nossa resposta formal. Primeiramente, convém rescrever a ação clássica no espaço euclidiano. Com este fim, escrevemos $t=-i \tau, \operatorname{com} \tau \in \mathbb{R}$. Segue, então, que $S=i S_{E}$, onde $S_{E}$ é a ação euclidiana dada por:

$$
S_{E}=\int_{\tau_{1}}^{\tau_{2}} d \tau\left[\frac{m}{2}\left(\frac{d x}{d \tau}\right)^{2}+V(x)\right] .
$$

Este resultado será empregado mais adiante quando reescrever a amplitude de transição no espaço euclidiano.

Queremos relacionar a amplitude de transição dada por (65) com a energia do estado fundamental do nosso sistema quântico. Para isso, é conveniente considerar $x_{2}=x_{1}, t_{1}=-T / 2$ e $t_{2}=T / 2$. Nesse caso, a amplitude de transição será dada por:

$$
\left\langle x_{1}, T / 2 \mid x_{1},-T / 2\right\rangle=\left\langle x_{1}|\exp (-i \hat{H} T)| x_{1}\right\rangle
$$

Vamos supor que o operador hamiltoniano $\hat{H}$ tenha um conjunto de auto-estados $\hat{H}|n\rangle=E_{n}|n\rangle$, ortonormais e completos, isto é, $\langle n \mid m\rangle=\delta_{n m}$ e $\sum_{n}|n\rangle\langle n|=\hat{1}$. Então:

$$
\begin{aligned}
\left(x_{1}, T / 2\left|x_{1},-T / 2\right\rangle\right. & =\sum_{n}\left\langle x_{1}|\exp (-i \hat{H} T)| n\right\rangle\left\langle n \mid x_{1}\right\rangle \\
& =\sum_{n} \exp \left(-i E_{n} T\right)\left\langle x_{1} \mid n\right\rangle\left\langle n \mid x_{1}\right\rangle .
\end{aligned}
$$

Integrando em $x_{1}$ e fazendo uso da relação de ortonormalidade:

$$
\int d x_{1}\left\langle x_{1} \mid n\right\rangle\left\langle n \mid x_{1}\right\rangle=\int d x_{1} \psi\left(x_{1}\right) \psi^{*}\left(x_{1}\right)=1
$$

obtemos

$$
\begin{aligned}
\sum_{n} \exp \left(-i E_{n} T\right) & =\int d x_{1}\left\langle x_{1}, T / 2 \mid x_{1},-T / 2\right\rangle \\
& =\int d x_{1} \int_{x(-T / 2)=x_{1}}^{x(T / 2)=x_{1}} D[x] \exp (i S[x]) \\
& =\int_{x(-T / 2)=x_{1}(T / 2)} D[x] \exp (i S[x])
\end{aligned}
$$

Para obtermos a versão euclidiana deste resultado, fazemos $T \rightarrow-i T_{E}$ e $S \rightarrow i S_{E}$, o que resulta em:

$$
\sum_{n} \exp \left(-E_{n} T_{E}\right)=\int_{x\left(-T_{E} / 2\right)=x\left(T_{E} / 2\right)} D[x] \exp \left(-S_{E}[x]\right) .
$$

Façamos a hipótese adicional de que o espectro de energia do operador hamiltoniano seja constituído por autovalores positivos e não-nulos. Neste caso, fazendo $T_{E} \rightarrow \infty$, vemos que o termo predominante é $\exp \left(-E_{0} T_{E}\right)$ e, conseqüentemente, podemos escrever:

$$
E_{0}=-\lim _{T_{E} \rightarrow \infty} \frac{1}{T_{E}} \ln \left(\int_{x\left(-T_{E} / 2\right)=x\left(T_{E} / 2\right)} D[x] \exp \left(-S_{E}[x]\right)\right)
$$




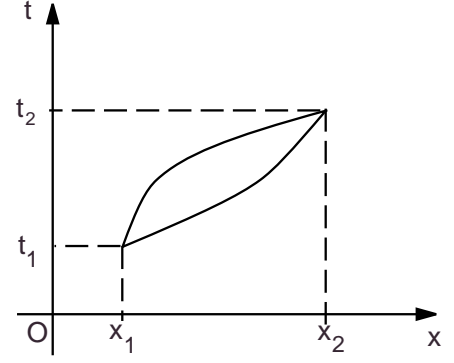

Figura 5. Curvas no espaço-tempo.

que é o resultado formal que buscávamos. Para uma certa classe de integrais de trajetória, ditas integrais gaussianas (veja o Apêndice A), a expressão acima pode ser explicitamente calculada. Vejamos dois exemplos que levam a integrais de trajetória gaussianas:

\section{O oscilador harmônico simples}

Considere-se primeiro, o oscilador harmônico de massa unitária. Seu potencial é dado por $V(x)=$ $(1 / 2) k x^{2}$. Integrando o termo de energia cinética por partes e fazendo uso do fato de que o movimento é periódico, podemos escrever a ação euclidiana na forma:

$$
S_{E}[x]=\frac{1}{2} \int_{-\frac{T_{E}}{2}}^{\frac{T_{E}}{2}} d \tau x(\tau)\left(-\frac{d^{2}}{d \tau^{2}}+\omega^{2}\right) x(\tau)
$$

que leva a uma integral de trajetória gaussiana. Conseqüentemente, a energia do estado fundamental do oscilador será dada por:

$$
\begin{aligned}
E_{0} & =-\lim _{T_{E} \rightarrow \infty} \frac{1}{T_{E}} \ln \left\{\int_{x\left(-T_{E} / 2\right)=x\left(T_{E} / 2\right)} D[x] \exp \left[-\frac{1}{2} \int_{-\frac{T_{E}}{2}}^{\frac{T_{E}}{2}} d \tau x(\tau)\left(-\frac{d^{2}}{d \tau^{2}}+\omega^{2}\right) x(\tau)\right]\right\} \\
& =-\lim _{T_{E} \rightarrow \infty} \frac{1}{T_{E}} \ln \left\{\left[\operatorname{det}\left(-\frac{d^{2}}{d \tau^{2}}+\omega^{2}\right)\right]^{-\frac{1}{2}}\right\}
\end{aligned}
$$

onde fizemos uso de A10) do Apêndice A. Observe-se que o determinante deve ser calculado no subespaço das curvas no espaço-tempo euclidiano, que obedecem à condição: $x\left(-T_{E} / 2\right)=x\left(T_{E} / 2\right)$.

\section{O campo escalar massivo}

O segundo exemplo que consideraremos mais adiante é dado pelo campo escalar real massivo $\varphi(\tau, \mathbf{x})$, em, por exemplo, $d+1$ dimensões, isto é: $\mathbf{x}=$ $\left(x_{1}, x_{2}, \ldots x_{d}\right)$, cuja densidade lagrangiana se escreve:

$$
-\mathcal{L}_{E}[\varphi]=\frac{1}{2}\left[\left(\frac{\partial \varphi}{\partial \tau}\right)^{2}+\left(\nabla_{(d)} \varphi\right)^{2}+m^{2} \varphi^{2}\right],
$$

onde $m$ é a massa da excitação associada ao campo quântico em questão. Novamente, uma integração por partes do termo cinético e o uso da condição $\varphi\left(-T_{E} / 2, \mathbf{x}\right)=\varphi\left(T_{E} / 2, \mathbf{x}\right)$ conduzem a:

$$
S_{E}[\varphi]=\frac{1}{2} \int d \tau d^{d} x \varphi(x)\left(-\partial_{E}^{2}+m^{2}\right) \varphi(x) .
$$

Em analogia ao caso do oscilador harmônico, a energia do estado fundamental do campo será dada por:

$$
E_{0}=-\lim _{T_{E} \rightarrow \infty} \frac{1}{T_{E}} \ln \left\{\int_{\varphi\left(-T_{E} / 2\right)=\varphi\left(T_{E} / 2\right)} D[\varphi]\right.
$$

$$
\begin{aligned}
& \left.\exp \left[-\frac{1}{2} \int d \tau d^{d} x \varphi(x)\left(-\partial_{E}^{2}+m^{2}\right) \varphi(x)\right]\right\} . \\
= & -\lim _{T_{E} \rightarrow \infty} \frac{1}{T_{E}} \ln \left\{\left[\operatorname{det}\left(-\partial_{E}^{2}+m^{2}\right)\right]^{-\frac{1}{2}}\right\},
\end{aligned}
$$

onde $\partial_{E}^{2}$ é o operador de D 'Alembert no espaço euclidiano:

$$
\partial_{E}^{2}:=\frac{\partial^{2}}{\partial \tau^{2}}+\nabla_{(d)}^{2},
$$

e o determinante deve ser calculado no espaço das funções que obedecem às condições dadas acima no tempo euclidiano e, possivelmente, condições de contorno impostas sobre algumas ou todas as variáveis espaciais, como ocorre no efeito Casimir. Nosso resultado é puramente formal. É natural, portanto, que nos perguntemos sobre como proceder para calcular tais determinantes e extrair deles resultados físicos. 


\section{V.1 O método da função zeta generaliza- da}

O método da função zeta generalizada é um método de calcular determinantes funcionais. Ele foi introduzido na teoria de campos no cálculo da energia associada ao efeito Casimir e ações efetivas por vários autores [9], [10]. Veja, também, a referência [11] quanto a uma apresentação mais detalhada. Aqui nos ateremos aos seus aspectos principais remetendo o leitor à literatura especializada para maiores detalhes.

Se $\hat{A}$ é um operador que satisfaz a equação de autovalores () do Apêndice $\mathrm{A}$, definimos a função zeta generalizada que lhe é associada por:

$$
\zeta(\hat{A} ; s):=\sum_{n}\left(\alpha_{n}\right)^{-s}, \quad(s \in \mathbb{C})
$$

onde $\alpha_{n}$ são os autovalores do operador $\hat{A}$ e $\Re s$ é suficientemente grande para dar sentido matemático a esta definição. De fato, é possível mostrar que $\sum_{n}\left(\alpha_{n}\right)^{-s}$ converge para $\Re s>d / p$, onde $d$ é a dimensão da variedade espaço-temporal compacta sem fronteiras em tela e $p$ é a ordem do operador (elíptico) $\hat{A}$ [11], [12]. A função zeta generalizada admite uma continuação analítica para uma função meromorfa. Se ela for analítica em $s=0$, podemos escrever para o determinante de $\hat{A}$ :

$$
\operatorname{det}(\hat{A}):=\exp \left[-\left.\frac{d}{d s} \zeta(\hat{A} ; s)\right|_{s=0}\right] .
$$

A motivação por trás dessa definição é facilmente percebida. De fato, suponhamos que haja um número finito de autovalores $\alpha_{n}$ de $\hat{A}$. Então, podemos escrever:

$$
\begin{aligned}
\operatorname{det}(\hat{A}) & =\Pi_{n} \alpha_{n} \\
& =\Pi_{n} \exp \left(\ln \alpha_{n}\right) \\
& =\exp \left(\sum_{n} \ln \alpha_{n}\right) \\
& =\exp \left(\sum_{n} \alpha^{-s} \ln \alpha_{n}\right)_{s=0} \\
& =\exp \left(-\sum_{n}\left(\frac{d \alpha_{n}^{-s}}{d s}\right)_{s=0}\right) \\
& =\exp \left(-\frac{d}{d s} \zeta(\hat{A} ; s)_{s=0}\right) .
\end{aligned}
$$

Entretanto, quando temos um número infinito de autovalores, mesmo que o produtório construído com os autovalores de $\hat{A}$ seja convergente, a relação entre o determinante e a função zeta pode não ser bem definida.
A razão é que talvez não seja possível escrever:

$$
\sum_{n}\left(\frac{d \alpha_{n}^{-s}}{d s}\right)_{s=0}=\frac{d}{d s}\left(\sum_{n} \alpha_{n}^{-s}\right)_{s=0},
$$

a menos que o somatório convirja absolutamente, invalidando a (82). Nesse caso, é necessário prescrever uma receita para o cálculo do determinante. A receita se lê: $(i)$ calcule os autovalores do operador $A$ para as condições de contorno em questão e construa a função zeta pertinente, $(i i)$ faça a extensão analítica da função zeta para o plano complexo $s$, ou pelo menos para um domínio que contenha a origem. Finalmente, ( $i i i)$ faça uso de (80). Se adotarmos o exposto acima, podemos escrever a energia do estado fundamental dada por (72) como:

$$
\begin{aligned}
E_{0} & =-\lim _{T_{E} \rightarrow \infty} \frac{1}{T_{E}} \ln (\operatorname{det} A)^{-1 / 2} \\
& =+\lim _{T_{E} \rightarrow \infty} \frac{1}{2 T_{E}} \ln (\operatorname{det} A) \\
& =-\lim _{T_{E} \rightarrow \infty} \frac{1}{2 T_{E}}\left[-\frac{d}{d s} \zeta(\hat{A} ; s)_{s=0}\right]
\end{aligned}
$$

onde naturalmente, o operador $\hat{A}$ deve estar relacionado com uma ação euclidiana que leve a integrais funcionais gaussianas encontradas nos dois exemplos precedentes. Vejamos, a seguir, como esta relação se aplica a duas situações concretas.

\section{V.1.1. O oscilador harmônico simples}

Como primeiro exemplo, consideremos o oscilador harmônico simples (que também pode ser visto como um exemplo de uma teoria de campos em $0+1$ dimensões). A energia do estado fundamental será dada por (74). Para calcular o determinante relevante, o primeiro passo é resolver a equação de autovalores:

$$
\left(-\frac{d^{2}}{d \tau^{2}}+\omega^{2}\right) \chi_{n}(\tau)=\alpha_{n} \chi_{n}(\tau),
$$

com a condição de contorno $\chi_{n}\left(-T_{E} / 2\right)=\chi_{n}\left(T_{E} / 2\right)$. Reconhecemos facilmente que a solução dessa equação de autovalores é proporcional a $\exp (i \kappa \tau)$. Ao fazermos uso da condição de contorno, os valores de $\kappa$ ficam restritos a múltiplos inteiros positivos e negativos de $2 \pi / T_{E}$. Segue que o espectro de autovalores de (85) é dado por:

$$
\alpha_{n}=\left(\frac{2 n \pi}{T_{E}}\right)^{2}+\omega^{2} ; n \in \mathbb{Z} .
$$

A função zeta será: 


$$
\begin{aligned}
\zeta\left(-\frac{d^{2}}{d \tau^{2}}+\omega^{2} ; s\right) & =\left(\frac{2 \pi}{T_{E}}\right)^{-2 s} \sum_{n \in \mathbb{Z}}\left[n^{2}+\left(\frac{T_{E} \omega}{2 \pi}\right)^{2}\right]^{-s} \\
& =\omega^{-2 s}+2\left(\frac{2 \pi}{T_{E}}\right)^{-2 s} \sum_{n \in \mathbb{N}}\left[n^{2}+\left(\frac{T_{E} \omega}{2 \pi}\right)^{2}\right]^{-s} .
\end{aligned}
$$

O somatório do lado direito pode ser identificado com uma função de Epstein unidimensional não-homogênea (veja o Apêndice B). Em particular, nossa função de Epstein tem a forma:

$$
E_{1}^{M^{2}}(z ; 1)=\sum_{n=1}^{\infty} \frac{1}{\left(n^{2}+M^{2}\right)^{z}}
$$

onde $\Re z>1 / 2$ e $M^{2}$ é uma constante positiva. A continuação analítica desta função é dada por (veja o Apêndice $\mathrm{B})$ :

$$
E_{1}^{M^{2}}(z ; 1)=-\frac{1}{2 M^{2 z}}+\frac{\sqrt{\pi}}{2 M^{2 z-1}} \frac{\Gamma\left(z-\frac{1}{2}\right)}{\Gamma(z)}+\frac{2 \sqrt{\pi}}{\Gamma(z)} \sum_{n \in \mathbb{N}}\left(\frac{n \pi}{M}\right)^{z-\frac{1}{2}} K_{-z+\frac{1}{2}}(2 \pi n M),
$$

onde $\Gamma(z)$ é a função gama de Euler e $K_{\nu}(z)$ é uma função de Bessel modificada de segunda espécie. Fazendo as adaptações necessárias, obtemos:

$$
\begin{aligned}
\zeta\left(-\frac{d^{2}}{d \tau^{2}}+\omega^{2} ; s\right)= & \frac{\omega T_{E}}{2 \pi^{\frac{1}{2}} \omega^{2 s}} \frac{\Gamma\left(s-\frac{1}{2}\right)}{\Gamma(s)} \\
& +\frac{2^{2} \pi^{s} \omega^{-2 s}}{\Gamma(s)}\left(\frac{2 \pi}{\omega T_{E}}\right)^{-s-\frac{1}{2}} \sum_{n \in \mathbb{N}} n^{s-\frac{1}{2}} K_{-s+\frac{1}{2}}\left(n \omega T_{E}\right) .
\end{aligned}
$$

Resta calcular a derivada da zeta em relação a $s$ e tomar o limite $s \rightarrow 0$. Essa tarefa torna-se simples quando recorremos a um resultado importante que envolve a função gama, a saber: quando $s \rightarrow 0$, a função $\Gamma(s)$ tende para $1 / s$. Se, por hipótese, $G(s)$ for uma função regular quando $s \rightarrow 0$, então,

$$
\begin{aligned}
\lim _{s \rightarrow 0} \frac{d}{d s}\left[\frac{G(s)}{\Gamma(s)}\right] & =\lim _{s \rightarrow 0}\left[\frac{1}{\Gamma(s)} \frac{d G(s)}{d s}-\frac{G(s)}{\Gamma^{2}(s)} \frac{d \Gamma(s)}{d s}\right] \\
& =\lim _{s \rightarrow 0}\left[\frac{1}{1 / s} \frac{d G(s)}{d s}-\frac{G(s)}{1 / s^{2}}\left(-1 / s^{2}\right)\right] \\
& =G(0) .
\end{aligned}
$$

Identificando $G(s)$ em (90) e calculando a derivada com (91), obtemos:

$$
G(0)=\frac{\omega T_{E}}{2 \pi^{\frac{1}{2}}} \Gamma\left(-\frac{1}{2}\right)+2\left(\frac{2 \pi}{\omega T_{E}}\right)^{-\frac{1}{2}} \sum_{n \in \mathbb{N}} n^{-\frac{1}{2}} K_{\frac{1}{2}}\left(n \omega T_{E}\right) .
$$

Substituindo este resultado em (84) e tomando o limite indicado, vemos que o segundo termo tende a zero, pois $T_{E}^{-1 / 2} K_{\frac{1}{2}}\left(n \omega T_{E}\right) \rightarrow 0$ neste limite. Portanto, a energia do estado fundamental do oscilador harmônico unidimensional é:

$$
E_{0}=\frac{\omega}{2}
$$

resultado sobejamente conhecido. O leitor perguntarse-á se vale a pena empregar técnica tão sofisticada para obter um resultado que pode ser obtido com técnicas mais familiares. A utilidade desta técnica está na sua aplicação em teoria quântica de campos, mas como muitas vezes sói acontecer, o oscilador harmônico simples (sempre ele) é o sistema ideal para ilustrar uma técnica nova de cálculo. O exemplo seguinte, não obstante sua simplicidade, diz respeito à teoria de campos. 


\section{V.1.2 Campo escalar sem massa em $1+1$ dimensões}

Como segundo exemplo, consideremos a energia de Casimir do campo escalar real $\varphi(\tau, x)$, sem massa, em $1+1$ dimensões. Como antes, consideramos que está submetido a condições de Dirichlet impostas em $x=0$ e $x=a$, isto é: $\varphi(\tau, 0)=\varphi(\tau, a)=0$. A equação de autovalores () se lê:

$$
-\partial_{E}^{2} \chi_{n}(\tau, x)=\alpha_{n} \chi_{n}(\tau, x) .
$$

As autofunções $\chi_{n}(\tau, x)$ de $-\partial_{E}^{2}$ formam uma base ortonormal no espaço das funções que satisfazem as condições de Dirichlet. É fácil ver que as autofunções ortonormalizadas são:

$$
\chi_{n}(\tau, x)=\frac{1}{\sqrt{a}} \frac{1}{\sqrt{2 \pi}} \sin \left(\frac{n \pi x}{a}\right) \exp (i \kappa \tau),
$$

e que os autovalores são dados por:

$$
\alpha_{n}=\kappa^{2}+\left(\frac{n \pi}{a}\right)^{2},
$$

com $\kappa \in \mathbb{R}$ e $n \in \mathbb{N}$. Segue que a função zeta é dada por:

$$
\zeta\left(-\partial_{E}^{2} ; s\right)=T_{E} \int_{-\infty}^{+\infty} \frac{d k}{2 \pi} \sum_{n \in \mathbb{N}}\left[k^{2}+\left(\frac{n \pi}{a}\right)^{2}\right]^{-s},
$$

onde fizemos uso da substituição $\sum \rightarrow\left(T_{E} / 2 \pi\right) d k$ para a parte contínua do espectro de autovalores dado por (96). Para dar prosseguimento ao cálculo, fazemos uso do resultado:

$$
\int \frac{d^{d} \mathbf{u}}{\left(\mathbf{u}^{2}+b^{2}\right)^{z}}=\pi^{\frac{d}{2}} \frac{\Gamma\left(z-\frac{d}{2}\right)}{\Gamma(z)}\left(b^{2}\right)^{-z+\frac{d}{2}},
$$

que pode ser obtido com o auxílio de dois resultados. Primeiro usamos:

$$
\int d^{d} \mathbf{u} f(u)=2 \frac{\pi}{\Gamma\left(\frac{d}{2}\right)} \int_{0}^{\infty} d u u^{d-1} f(u)
$$

onde $u=|\mathbf{u}|$ e depois [5]:

$\int_{0}^{\infty} d x x^{\mu-1}\left(x^{2}+c^{2}\right)^{\nu-1}=\frac{c^{\mu+2 \nu-2}}{2} B\left(\frac{\mu}{2} ; 1-\nu-\frac{\mu}{2}\right)$,

onde:

$$
B(x, y)=\frac{\Gamma(x) \Gamma(y)}{\Gamma(x+y)},
$$

e $\Re(\nu+\mu / 2)<1$ e $\Re \mu>0$. Fazendo uso de (98) em (97), obtemos:

$$
\zeta\left(-\partial_{E}^{2} ; s\right)=\frac{T_{E}}{2 \pi} \pi^{\frac{1}{2}} \frac{\Gamma\left(s-\frac{1}{2}\right)}{\Gamma(s)}\left(\frac{\pi}{a}\right)^{-2 s+1} \sum_{n \in \mathbb{N}} n^{-2 s+1} .
$$

Observemos que, como no caso do oscilador, a função zeta é da forma $G(s) / \Gamma(s)$ e que o somatório no lado direito de (102) é a função zeta de Riemann $\zeta_{R}(2 s-1)$. Procedendo como antes, i.e.: calculando a derivada com (91) e substituindo em (84), obtemos o resultado final:

$$
E_{0}(a)=-\frac{\pi}{24 a}
$$

onde fizemos uso do resultado $\zeta_{R}(-1)=-1 / 12$, que pode ser obtido fazendo $z=-1$ na fórmula de reflexão $[5]$ :

$$
2^{z} \Gamma(1-z) \zeta_{R}(1-z) \sin \left(\frac{z \pi}{2}\right)=\pi^{1-z} \zeta_{R}(z) .
$$

O resultado obtido para a energia do vácuo do campo escalar real sem massa em $1+1$ é o mesmo que obtivemos anteriormente com outros métodos. O sinal negativo indica que os pontos de Dirichlet atraem-se mutuamente.

\section{O método da função de Green}

Um segundo método de cálculo do determinante e, conseqüentemente, da energia do estado fundamental, é o método da função de Green. De fato, como:

$$
\ln [\operatorname{det}(\hat{A})]=\operatorname{Tr}[\ln (\hat{A})],
$$

onde o símb olo $\operatorname{Tr}$ significa traço, ou seja, a soma dos autovalores do operador $\hat{A}$ em questão. Na representação em que este é diagonal, podemos escrever [veja (77)]:

$$
E_{0}=\lim _{T_{E} \rightarrow \infty} \frac{1}{2 T_{E}} \operatorname{Tr}[\ln (\hat{A})]
$$

onde $\hat{A}$ é o operador de interesse. Com isso eliminamos o expoente $-1 / 2$ dentro do determinante em (77) e ficamos com o logaritmo do operador de interesse. A idéia por trás do método é expressar o traço do operador $\hat{A}$ em termos da função de Green que lhe é associada. Passemos aos exemplos.

\section{VI.1 O oscilador harmônico sim- ples}

Comecemos com o oscilador harmônico simples. Nesse caso:

$$
E_{0}=\lim _{T_{E} \rightarrow \infty} \frac{1}{2 T_{E}} \operatorname{Tr}\left[\ln \left(-\frac{d^{2}}{d \tau^{2}}+\omega^{2}\right)\right] .
$$

Derivando esta relação em relação a $\omega^{2}$, temos:

$$
\frac{\partial E_{0}}{\partial \omega^{2}}=\lim _{T_{E} \rightarrow \infty} \frac{1}{2 T_{E}} \operatorname{Tr}\left(-\frac{d^{2}}{d \tau^{2}}+\omega^{2}\right)^{-1},
$$


onde agora o traço é sobre o inverso do operador em questão. O operador inverso na representação $\tau$ é a função de Green e satisfaz a equação diferencial:

$$
\left(-\frac{d^{2}}{d \tau^{2}}+\omega^{2}\right) G\left(\tau, \tau^{\prime} ; \omega\right)=\delta\left(\tau-\tau^{\prime}\right),
$$

onde,

$$
G\left(\tau, \tau^{\prime} ; \omega\right)=\left(-\frac{d^{2}}{d \tau^{2}}+\omega^{2}\right)^{-1} \delta\left(\tau-\tau^{\prime}\right),
$$

ou,

$$
G\left(\tau, \tau^{\prime} ; \omega\right)=\left\langle\tau|\hat{G}| \tau^{\prime}\right\rangle .
$$

Como queremos o traço da função de Green, fazemos $\tau=\tau^{\prime}$ e escrevemos:

$$
\frac{\partial E_{0}}{\partial \omega^{2}}=\lim _{T_{E} \rightarrow \infty} \frac{1}{2 T_{E}} \int_{-\frac{T_{E}}{2}}^{\frac{T_{E}}{2}} d \tau G(\tau, \tau ; \omega) .
$$

Vemos então que para prosseguir devemos, em primeiro lugar, resolver o problema do cálculo explícito da função de Green do oscilador harmônico simples, o que pode ser feito com o método da transformada de Fourier. Substituindo em (109) as representações de Fourier:

$$
G\left(\tau, \tau^{\prime} ; \omega\right)=\int_{-\infty}^{+\infty} \frac{d \nu}{2 \pi} \exp \left[i\left(\tau-\tau^{\prime}\right) \nu\right] g(\nu ; \omega),
$$

e,

$$
\delta\left(\tau-\tau^{\prime}\right)=\int_{-\infty}^{+\infty} \frac{d \nu}{2 \pi} \exp \left[i\left(\tau-\tau^{\prime}\right) \nu\right],
$$

obtemos:

$$
\left(\nu^{2}+\omega^{2}\right) g(\nu ; \omega)=1 \text {. }
$$

Conseqüentemente:

$$
G\left(\tau-\tau^{\prime} ; \omega\right)=\int_{-\infty}^{+\infty} \frac{d \nu}{2 \pi} \frac{\exp \left[i\left(\tau-\tau^{\prime}\right) \nu\right]}{\nu^{2}+\omega^{2}} .
$$

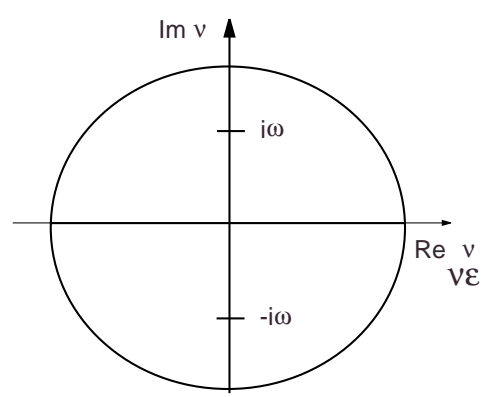

Figura 6. O plano complexo $\nu$.

O integrando apresenta dois pólos simples em $\nu= \pm i \omega$, veja a Fig. 6.

Se $\tau>\tau^{\prime}, \quad$ como $\exp \left[i\left(\tau-\tau^{\prime}\right) \nu\right]=$ $\exp \left[i\left(\tau-\tau^{\prime}\right) \Re \nu-\left(\tau-\tau^{\prime}\right) \Im \tau\right]$, fechamos o contorno no plano $\Im \nu>0$ no sentido anti-horário e usamos o teorema de Cauchy, obtendo:

$$
G_{+}\left(\tau-\tau^{\prime} ; \omega\right)=\frac{1}{2 \omega} \exp \left[-\left(\tau-\tau^{\prime}\right) \omega\right],
$$

e se $\tau<\tau^{\prime}$, fechamos o contorno no plano $\Im \nu<0$ no sentido horário, obtendo nesse caso:

$$
G_{-}\left(\tau-\tau^{\prime} ; \omega\right)=\frac{1}{2 \omega} \exp \left[-\left(\tau^{\prime}-\tau\right) \omega\right] .
$$

A função de Green correta é $(\theta(\tau)$ é a função degrau de Heaviside):

$$
\begin{aligned}
G\left(\tau-\tau^{\prime} ; \omega\right) & =\frac{1}{2 \omega}\left[\theta\left(\tau-\tau^{\prime}\right) \exp \left[-\left(\tau-\tau^{\prime}\right) \omega\right]+\theta\left(\tau^{\prime}-\tau\right) \exp \left[-\left(\tau^{\prime}-\tau\right) \omega\right]\right] \\
& =\frac{1}{2 \omega} \exp \left(-\left|\tau-\tau^{\prime}\right| \omega\right)
\end{aligned}
$$

Como queremos o traço, escrevemos $G(0 ; \omega)=1 /(2 \omega)$ e portanto:

$$
\begin{aligned}
\frac{\partial E_{0}}{\partial \omega^{2}} & =\frac{1}{2 \omega} \lim _{T_{E} \rightarrow \infty} \frac{1}{2 T_{E}} \int_{-\frac{T_{E}}{2}}^{\frac{T_{E}}{2}} d \tau \\
& =\frac{1}{4 \omega} .
\end{aligned}
$$

Logo, escrevendo $\partial E_{0} / \partial \omega^{2}=\left(\partial \omega / \partial \omega^{2}\right)\left(\partial E_{0} / \partial \omega\right)$, temos:

$$
\frac{\partial E_{0}}{\partial \omega}=\frac{1}{2} .
$$

Integrando em relação a $\omega$, obtemos finalmente:

$$
E_{0}=\frac{\omega}{2}+C,
$$

onde $C$ é uma constante de integração que podemos escolher com sendo nula, recuperando deste modo o resultado familiar da mecânica quântica usual. 


\section{VI.1.1 Campo escalar em $3+1$ dimensões}

Como segundo exemplo, consideremos uma vez mais o campo escalar real $\varphi$, mas desta feita, com um pouco mais de realidade, em $3+1$ dimensões. Para que possamos aplicar a técnica da função de Green ao cálculo do determinante bosônico, admitiremos a hipótese de que o campo escalar é massivo. A massa $m$ associada ao quantum do campo fará o papel da freqüência $\omega$ no caso do oscilador. Ao final, se nos for conveniente, poderemos tomar o limite de massa nula. A ação euclidiana se escreve então:

$$
S_{E}[\varphi]=\frac{1}{2} \int d \tau d^{3} x \varphi(\tau, \mathbf{x})\left(-\partial_{E}^{2}+m^{2}\right) \varphi(\tau, \mathbf{x}) .
$$

Suponhamos que, em $z=0$ e $z=a$, o campo tenha que satisfazer condições de contorno de Dirichlet. Também neste caso, a energia do estado fundamental será dada por:

$$
E_{0}(a)=\lim _{T_{E} \rightarrow \infty} \frac{1}{2 T_{E}} \operatorname{Tr}\left[\ln \left(-\partial_{E}^{2}+m^{2}\right)\right],
$$

estando subentendido que o traço deve ser calculado no espaço das funções que obedecem às condições de Dirichlet, além da condição $\varphi\left(-T_{E} / 2, \mathbf{x}\right)=$ $\varphi\left(T_{E} / 2, \mathbf{x}\right)$. Repetindo os passos que demos no caso do oscilador harmônico, derivamos a energia $E_{0}(a)$ em relação à massa ao quadrado para obter:

$$
\frac{\partial E_{0}(a)}{\partial m^{2}}=\lim _{T_{E} \rightarrow \infty} \frac{1}{2 T_{E}} \operatorname{Tr}\left(-\partial_{E}^{2}+m^{2}\right)^{-1} .
$$

Como antes:

$$
\left(-\partial_{E}^{2}+m^{2}\right) G\left(\tau, \mathbf{x}, \tau^{\prime}, \mathbf{x}^{\prime}\right)=\delta\left(\tau-\tau^{\prime}\right) \delta\left(\mathbf{x}-\mathbf{x}^{\prime}\right)
$$

o que significa que na representação de coordenadas:

$$
G\left(\tau, \mathbf{x}, \tau^{\prime}, \mathbf{x}^{\prime}\right)=\left(-\partial_{E}^{2}+m^{2}\right)^{-1} \delta\left(\tau-\tau^{\prime}\right) \delta\left(\mathbf{x}-\mathbf{x}^{\prime}\right),
$$

ou ainda:

$$
G\left(\tau, \mathbf{x}, \tau^{\prime}, \mathbf{x}^{\prime}\right)=\left\langle\tau, \mathbf{x}\left|\left(-\partial_{E}^{2}+m^{2}\right)^{-1}\right| \tau^{\prime}, \mathbf{x}^{\prime}\right\rangle .
$$

A geometria do sistema que estamos estudando permite que a função de Green possa ser construída com o método das imagens. De fato, para condições de contorno de Dirichlet é possível mostrar que [15]:

$$
\begin{gathered}
G\left(\tau, \mathbf{x}, \tau^{\prime}, \mathbf{x}^{\prime}\right)=\sum_{n \in \mathbb{Z}} G_{0}\left(\tau-\tau^{\prime}, \mathbf{x}-\mathbf{x}^{\prime}+2 a n \hat{\mathbf{u}}\right)- \\
\sum_{n \in \mathbb{Z}} G_{0}\left(\tau+\tau^{\prime}, \mathbf{x}+\mathbf{x}^{\prime}+2 a n \hat{\mathbf{u}}\right),
\end{gathered}
$$

onde $G_{0}\left(\tau-\tau^{\prime}, \mathbf{x}-\mathbf{x}^{\prime}\right)$ é o propagador da partícula escalar livre e $\hat{\mathbf{u}}=(0,0,0,1)$. O primeiro somatório representa a propagação entre $\left(\tau^{\prime}, \mathbf{x}^{\prime}\right)$ e $(\tau, \mathbf{x})$ com um número par de reflexões; o segundo somatório (e o sinal negativo) representa a propagação entre $\left(\tau^{\prime}, \mathbf{x}^{\prime}\right)$ e $(\tau, \mathbf{x})$ com um número ímpar de reflexões, veja a

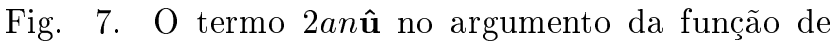
Green livre localiza a posição das imagens. Mais adiante mostraremos que, no cálculo do traço, o segundo somatório não depende da distância $a$ e, portanto, pode ser descartado. No momento, porém, deixemos este ponto de lado e concentremos nossa atenção no propagador $G_{0}\left(\tau-\tau^{\prime}, \mathbf{x}-\mathbf{x}^{\prime}\right)$. Como mostrado no Apêndice $\mathrm{C}$, este propagador pode ser calculado com o método do tempo próprio introduzido por J. Schwinger em um famoso artigo publicado em 1951 [16]. O resultado se lê:

$$
G_{0}\left(\tau-\tau^{\prime}, \mathbf{x}-\mathbf{x}^{\prime}\right)=\frac{m}{4 \pi^{2}|\Delta|} K_{1}(m|\Delta|)
$$

onde $K_{1}(m|\Delta|)$ é uma função de Bessel modificada com $\Delta^{2}:=\left(\tau-\tau^{\prime}\right)^{2}+\left(\mathbf{x}-\mathbf{x}^{\prime}\right)^{2}$. Fisicamente, esse resultado pode ser interpretado como descrevendo a propagação de uma partícula escalar livre em quatro dimensões euclidianas na representação de coordenadas. Passemos, agora, ao cálculo da energia de Casimir do campo em questão.

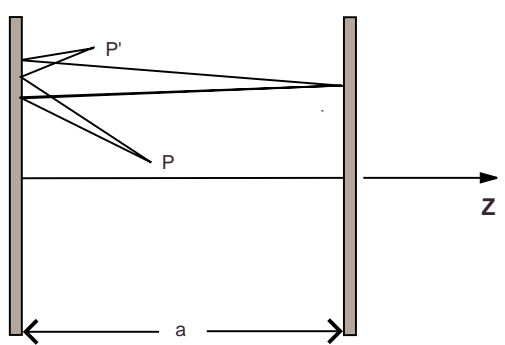

Figura 7. A geometria simples das superfícies de Dirichlet paralelas permite o uso do método das imagens no cálculo da função de Green relevante.

\section{VI.1.2 A energia de Casimir do campo escalar em $3+1$ dimensões}

Voltemos ao problema original e construamos a função de Green para as condições de Dirichlet. Substituindo (130) em (129), temos:

$$
\begin{aligned}
G\left(\tau, \mathbf{x}, \tau^{\prime}, \mathbf{x}^{\prime}\right) & =\sum_{n \in \mathbb{Z}} G_{0}\left(\tau-\tau^{\prime}, \mathbf{x}-\mathbf{x}^{\prime}+2 a n \hat{\mathbf{u}}\right) \\
& =\frac{m}{4 \pi^{2}} \sum_{n \in \mathbb{Z}} \frac{K_{1}(m|\Delta+2 a n \hat{\mathbf{u}}|)}{|\Delta+2 a n \hat{\mathbf{u}}|}
\end{aligned}
$$


Como queremos o propagador no mesmo ponto do espaço-tempo euclidiano, segue que:

$$
G(\tau, \mathbf{x}, \tau, \mathbf{x})=\frac{(a m)}{8 \pi^{2} a^{2}} \sum_{n \in \mathbb{Z}-\{0\}} \frac{1}{|n|} K_{1}(2 m a|n|),
$$

em que o termo (divergente) correspondente a $n=0$ foi excluído e o propagador, portanto, renormalizado.
A subtração do termo $n=0$ corresponde à subtração de (129) ou (131) do propagador da partícula livre na ausência de placas. Outro modo de justificar essa subtração é reconhecer que esse termo não depende da distância entre as placas. Substituindo este resultado na (125), obtemos:

$$
\begin{aligned}
\frac{\partial E_{0}}{\partial m^{2}} & =\lim _{T_{E} \rightarrow \infty} \frac{1}{2 T_{E}} \int_{-\frac{T_{E}}{2}}^{\frac{T_{E}}{2}} d \tau \int d x \int d y \int_{0}^{a} d z G(\tau, \mathbf{x}, \tau, \mathbf{x}) \\
& =A \frac{(a m)}{8 \pi^{2} a} \sum_{n \in \mathbb{N}} \frac{1}{n} K_{1}(2 \operatorname{man}),
\end{aligned}
$$

onde $A$ é a área da superfície sobre a qual as condições de Dirchlet vigoram e um fator 2 foi introduzido ao fazermos a troca $\mathbb{Z}-\{0\}$ por $\mathbb{N}$. Para integrar (133), fazemos uso da relação [5]:

$$
\left(\frac{d}{z d z}\right)^{j}\left[z^{\nu} K_{\nu}(z)\right]=(-1)^{j} z^{\nu-j} K_{\nu-j}(z),
$$

com a qual podemos mostrar que:

$$
K_{1}(2 a m n)=-\frac{1}{a m n} \frac{d}{d m^{2}}\left[m^{2} K_{2}(2 a m n)\right] .
$$

Segue então que:

$$
\frac{1}{A} \frac{\partial E_{0}}{\partial m^{2}}=-\frac{1}{8 \pi^{2} a} \frac{\partial}{\partial m^{2}} \sum_{n \in \mathbb{N}} \frac{m^{2}}{n^{2}} K_{2}(2 a m n) .
$$

A integração em $m^{2}$ é imediata e leva a:

$$
\frac{E_{0}(a)}{A}=-\frac{(a m)^{2}}{8 \pi^{2} a^{3}} \sum_{n \in \mathbb{N}} \frac{1}{n^{2}} K_{2}(2 a m n),
$$

onde a constante de integração fica determinada pelo fato de que, no limite extremo em que a massa da excitação do campo vai ao infinito, a energia de Casimir deve ser nula. Essa condição combinada com o fato de que $K_{\nu}(z) \rightarrow 0$ para $z \rightarrow \infty$ (veja a (138) abaixo) nos leva a concluir que a constante de integração é zero.

No limite em que $a m \gg 1$, apenas o termo correspondente a $n=1 \mathrm{em} \mathrm{(137)} \mathrm{é} \mathrm{significativo,} \mathrm{e} \mathrm{fazendo}$ uso de:

$$
K \nu(z) \approx\left(\frac{\pi}{2 z}\right)^{1 / 2} \exp (-z), \quad z \rightarrow \infty,
$$

com $z=2 a m$, obtemos:

$$
\frac{E_{0}(a)}{A} \approx-\frac{1}{16 a^{3}}\left(\frac{a m}{\pi}\right)^{3 / 2} \exp (-2 a m),
$$

de acordo com a literatura [21].
Outro limite importante é o limite de massa nula, o qual pode ser obtido se fizermos o uso da relação [5]:

$$
\lim _{z \rightarrow 0} K_{n}(z) \approx(n-1) ! 2^{n-1} z^{-n}
$$

válida para $n \in \mathbb{N}$. Segue, então, que:

$$
\frac{E_{0}(a)}{A} \approx-\frac{1}{16 \pi^{2} a^{3}} \sum_{n \in \mathbb{N}} \frac{1}{n^{4}} .
$$

A soma $\sum_{n \in \mathbb{N}} n^{-4}$ define a função zeta de Riemann $\zeta_{R}(4)$. Seu valor numérico é [5]:

$$
\zeta_{R}(4)=\frac{\pi^{4}}{90}
$$

Portanto, a energia de Casimir por unidade de área no limite de massa zero será:

$$
\frac{E_{0}(a)}{A} \approx-\frac{\pi^{2}}{1440 a^{3}}
$$

Esse resultado também concorda perfeitamente com o encontrado na literatura [21]. Convém lembrar que a multiplicação desse resultado por um fator 2 reproduz o efeito Casimir eletromagnético para placas paralelas perfeitamente condutoras separadas por uma distância a. O fator 2 toma conta dos graus de liberdade de polarização do campo eletromagnético. Entretanto, isso acontece somente para a geometria simples das placas paralelas, para outras geometrias a simulação do campo eletromagnético por um campo escalar real não é tão simples assim.

Resta justificar a razão pela qual a parcela do propagador correspondente a um número ímpar de reflexões não contribui para o efeito Casimir [17].

Em $3+1$ o cálculo do traço leva ao cálculo da integral: 


$$
I=-\int d x \int d y \int_{0}^{a} d z \sum_{n \in \mathbb{Z}} G_{0}(2 \tau, 2 x, 2 y, 2 z+2 a n \hat{\mathbf{u}}) .
$$

Introduzindo-se a variável $\xi:=z+n a, n \in \mathbf{N}$, a integral pode ser rescrita como:

$$
I=-\int d x \int d y \sum_{n \in \mathbb{Z}} \int_{n a}^{(n+1) a} d \xi G_{0}(2 \tau, 2 x, 2 y, 2 \xi) .
$$

Mas é fácil ver que:

$$
\begin{aligned}
\sum_{n \in \mathbb{Z}} \int_{n a}^{(n+1) a} d \xi G_{0}(2 \tau, 2 x, 2 y, 2 \xi)= & \int_{0}^{a} d \xi \ldots+\int_{a}^{2 a} d \xi \ldots+\int_{2 a}^{3 a} d \xi \ldots \\
& +\int_{-a}^{0} d \xi \ldots+\int_{-2 a}^{-a} d \xi \ldots+\int_{-3 a}^{-2 a} d \xi \ldots \\
= & \int_{-\infty}^{+\infty} d \xi G_{0}(2 \tau, 2 x, 2 y, 2 \xi)
\end{aligned}
$$

e, portanto, esta integral não dependerá da distância $a$ entre as superfícies planas.

\section{Considerações finais e in- dicações bibliográficas}

Neste trabalho, procuramos contribuir para a literatura pedagógico-científica em língua portuguesa de modo geral e para aquela que concerne aos efeitos da vácuo quântico em particular, apresentando ao leitor alguns dos métodos de cálculo da energia de Casimir. Começamos discutindo o conceito de energia de Casimir e salientamos a importância da sua regularização. Depois mostramos, por meio do formalismo canônico, que a energia de Casimir é uma característica das teorias quânticas de campo relativísticas submetidas a condições de contorno. A seguir, apresentamos os métodos de cálculo da energia de Casimir, começando com dois métodos diretos: o método do corte e o método da regularização na rede. Depois introduzimos a noção mais sofisticada da energia do vácuo como o logaritmo de um determinante funcional bosônico e calculamos essa quantidade por meio do método da função zeta generalizada e depois por meio da função de Green. Em todos os casos discutimos exemplos cuja simplicidade facilita a compreensão do método em questão. Como já mencionado anteriormente, os quatro métodos discutidos aqui não esgotam o arsenal de métodos utilizados no cálculo da energia de Casimir, mas são significativos.

Finalmente, gostaríamos de concluir dando ao leitor algumas indicações bibliográficas. Parte da literatura sobre o efeito Casimir é apresentada em um levantamento bibliográfico recente que se deve a Lamoreaux [18]. Ao leitor interessado em aprofundar seus conhecimentos, sem por isso ser aterrado por uma montanha de detalhes técnicos, recomendamos o artigo de Elizalde e Romeo [7] e a monografia de Mostepanenko e Trunov [19], que contém uma visão geral completa e mais avançada do efeito Casimir e suas aplicações a diversos campos da física, especialmente à cosmologia e à física das partículas elementares. Dos mesmo autores, o leitor desejoso de um aprendizado mais técnico e profundo poderá consultar a referência [20]. Neste sentido, o artigo de revisão de Pluniem et al. [21], embora não tão recente, é também recomendado. Progressos recentes, experimentais e teóricos, são apresentados por Bordag, Mohideen e Mostepanenko em [22]. Na referência [23], além do efeito Casimir, o leitor encontrará discussões sobre outros tópicos relacionados com a física do vácuo quântico, como por exemplo, as forças de van der Waals de dispersão e sua relação com o efeito Casimir. Todas essas referências contêm extensas coleções de referências bibliográficas sobre o tema. $\mathrm{O}$ artigo original de Casimir pode ser lido em [24], veja também [25] quanto a uma versão com notação atualizada. Uma tradução para o português do artigo original de Casimir pode ser encontrada no apêndice da referência [26], que traz também uma sinopse (em português) da evolução do conceito de vácuo ao longo da história. 


\section{Agradecimentos}

Os autores agradecem aos professores M. V. CougoPinto, C. Farina e F. C. Santos pela leitura atenta do manuscrito original e sugestões. J. J. Passos Sobrinho agradece ao $\mathrm{CNPq}$ pelo auxílio recebido.

\section{Apêndice A: integrais gaussianas}

A energia do estado fundamental dada por (72) pode ser calculada de modo exato nos casos em que a integral funcional é da forma dita gaussiana em analogia com as integrais gaussianas ordinárias. Vejamos então, sem muito rigor matemático, e sim com argumentos de plausibilidade, como integrais funcionais gaussianas podem ser explicitamente calculadas.

Considere-se a integral gaussiana ordinária:

$$
\int_{-\infty}^{+\infty} d y \exp \left(-\frac{1}{2} a y^{2}\right)=(2 \pi)^{\frac{1}{2}} a^{-\frac{1}{2}}, \quad a>0
$$

Queremos generalizar este resultado e mostrar que:

$$
\int_{-\infty}^{+\infty} d y_{1} \ldots d y_{N} \exp \left(-\frac{1}{2}\langle Y|\hat{A}| Y\rangle\right)=(2 \pi)^{\frac{N}{2}}(\operatorname{det} \hat{A})^{-\frac{1}{2}}
$$

onde $|Y\rangle$ é um vetor pertencente a um espaço vetorial real de dimensão finita,

$$
|Y\rangle=\left(\begin{array}{c}
y_{1} \\
\vdots \\
y_{N}
\end{array}\right)
$$

com $y_{i} \in \mathbb{R}$. O operador $\hat{A}$ é um operador definido positivo representado por uma matriz real e simétrica, isto é: seus autovalores são números reais, positivos e nenhum deles é nulo. Suponhamos que o operador $\hat{A}$ possua um conjunto ortonormal completo de autovetores $\left|\alpha_{n}\right\rangle$ tal que:

$$
\hat{A}\left|\alpha_{n}\right\rangle=\alpha_{n}\left|\alpha_{n}\right\rangle,
$$

$\operatorname{com}\left\langle\alpha_{n} \mid \alpha_{m}\right\rangle=\delta_{m n} \mathrm{e}$,

$$
\sum_{n=1}^{N}\left|\alpha_{n}\right\rangle\left\langle\alpha_{n}\right|=\hat{1}
$$

Podemos expandir o vetor $|Y\rangle$ na base formada pelos autovetores de $\hat{A}$ :

$$
|Y\rangle=\sum_{n=1}^{N} c_{n}\left|\alpha_{n}\right\rangle
$$

Segue que podemos escrever:

$$
\begin{gathered}
\langle Y|\hat{A}| Y\rangle=\sum_{m=1}^{N} \sum_{n=1}^{N} c_{m} c_{n}\left\langle\alpha_{m}|\hat{A}| \alpha_{n}\right\rangle \\
=\sum_{n=1}^{N} c_{n}^{2} \alpha_{n} .
\end{gathered}
$$

Observando que a transformação de base é linear e que seu jacobiano vale a unidade, escrevemos:

$$
\begin{gathered}
\int_{-\infty}^{+\infty} d y_{1} \ldots d y_{N} \exp \left(-\frac{1}{2}\langle Y|\hat{A}| Y\rangle\right) \\
=\int_{-\infty}^{+\infty} d c_{1} \ldots d c_{N} \exp \left(-\frac{1}{2} \sum_{n=1}^{N} c_{n}^{2} \alpha_{n}\right) \\
=(2 \pi)^{\frac{N}{2}}\left(\Pi_{n=1}^{N} \alpha_{n}\right)^{-\frac{1}{2}} \\
=(2 \pi)^{\frac{N}{2}}(\operatorname{det} \hat{A})^{-\frac{1}{2}}
\end{gathered}
$$

que é o resultado que nos propusemos obter. Quando o vetor $|Y\rangle$ tem uma infinidade contínua de componentes, a equação acima torna-se uma integral funcional gaussiana, que formalmente representamos por:

$$
\int D[Y] \exp \left(-\frac{1}{2}\langle Y|\hat{A}| Y\rangle\right)(\operatorname{det} \hat{A})^{-\frac{1}{2}}
$$

É possível mostrar que:

$$
\int D[Y] \exp \left(-\frac{1}{2}\langle Y|\hat{A}| Y\rangle\right)=(\operatorname{det} \hat{A})^{-\frac{1}{2}},
$$

onde o fator $(2 \pi)^{\frac{N}{2}}$, que diverge no limite $N \rightarrow \infty$, é levado em conta na medida da integral funcional. Tomando o logaritmo de (A9) obtemos

$$
\ln \int D[Y] \exp \left(-\frac{1}{2}\langle Y|\hat{A}| Y\rangle\right)=\ln (\operatorname{det} \hat{A})^{-\frac{1}{2}}
$$

\section{Apêndice B: funções de Epstein}

As funções de Epstein multidimensionais inomogêneas [27] (veja também Elizalde et al. [11] para uma apresentação moderna) são definidas por: 


$$
E_{N}^{M^{2}}\left(z ; a_{1}, a_{2}, \ldots a_{N}\right)=\sum_{n_{1}, n_{2}, . . n_{N}=1}^{\infty}\left(a_{1} n_{1}^{2}+a_{2} n_{2}^{2}+\cdots+a_{N} n_{N}^{2}+M^{2}\right)^{-z},
$$

for $\Re z>N / 2$ and $a_{1}, a_{2}, \ldots a_{N}>0$. Esta função possui uma continuação analítica para uma função meromórfica que para $N=1$ é dada por:

$$
\begin{gathered}
E_{1}^{M^{2}}(z ; a)=-\frac{1}{2 M^{2 z}}+\left(\frac{\pi}{a}\right)^{1 / 2} \frac{1}{2 M^{2 z-1} \Gamma(z)} \\
\times\left[\Gamma\left(z-\frac{1}{2}\right)+4 \sum_{n=1}^{\infty} \frac{a^{(1-2 z) / 4}}{(\pi M n)^{(1 / 2)-z}} K_{(1 / 2)-z}\left(\frac{2 \pi M n}{a^{1 / 2}}\right)\right] .
\end{gathered}
$$

Para prová-lo, consideremos inicialmente:

$$
\begin{gathered}
E_{1}^{M^{2}}(z ; a)=\sum_{n=1}^{\infty}\left(a n^{2}+M^{2}\right)^{-z} \\
=a^{-z} \sum_{n=1}^{\infty}\left(n^{2}+C^{2}\right)^{-z}
\end{gathered}
$$

onde $C^{2} \equiv M^{2} / a$. Com a representação integral da função gama de Euler:

$$
\Gamma(z) \alpha^{-z}=\int_{0}^{\infty} d t t^{z-1} \exp (-\alpha t) ; \quad \Re z>0,
$$

podemos escrever:

$$
E_{1}^{M^{2}}(z ; a)=\frac{a^{-z}}{\Gamma(z)} \int_{0}^{\infty} d t t^{z-1} \exp \left(-C^{2} t\right) \sum_{n=1} \exp \left(-n^{2} t\right)
$$

A seguir introduzimos a fórmula de Poisson [28]:

$$
\sum_{n=-\infty}^{\infty} \exp \left(-n^{2} \pi t\right)=\sqrt{\frac{1}{t}} \sum_{n=-\infty}^{\infty} \exp \left(-n^{2} \pi / t\right)
$$

a qual, fazendo a substituição $\pi t \rightarrow t$, reescrevemos aqui de forma mais conveniente:

$$
\sum_{n=1}^{\infty} \exp \left(-n^{2} \pi t\right)=-\frac{1}{2}+\frac{1}{2 \sqrt{t}}+\frac{1}{\sqrt{t}} \sum_{n=1}^{\infty} \exp \left[\left(-n^{2} \pi\right) / t\right]
$$

Segue então que podemos escrever para $E_{1}^{M^{2}}(z ; a)$ a expressão:

$$
\begin{aligned}
E_{1}^{M^{2}}(z ; a)= & -\frac{1}{2} \frac{a^{-z}}{\Gamma(z)} \int_{0}^{\infty} d t t^{z-1} \exp \left(-C^{2} t\right)+\frac{1}{2} \frac{a^{-z}}{\Gamma(z)} \sqrt{\pi} \int_{0}^{\infty} d t t^{z-1 / 2-1} \exp \left(-C^{2} t\right) \\
& +\frac{a^{-z}}{\Gamma(z)} \sqrt{\pi} \sum_{n=1}^{\infty} \int_{0}^{\infty} d t t^{z-1 / 2-1} \exp \left(-C^{2} t\right) \exp \left[\left(-n^{2} \pi\right) / t\right] .
\end{aligned}
$$

Recorrendo-se novamente à definição da função gama de Euler, o primeiro termo reduz-se à $-1 / 2 M^{2 z}$, e o segundo a:

$$
\frac{1}{2} \sqrt{\frac{\pi}{a}} \frac{\Gamma(z-1 / 2)}{\Gamma(z)} \frac{1}{M^{2 z+1}}
$$

O terceiro termo pode ser tratado com a representação integral da função de Bessel modificada do segundo tipo, veja (C9) no Apêndice C. O terceiro termo é então:

$$
2\left(\frac{n \pi a^{1 / 2}}{M}\right)^{z-1 / 2} K_{z-1 / 2}\left(\frac{2 n \pi M}{\sqrt{a}}\right) .
$$

Coletando todos os termos e rearranjando-os de 
modo conveniente, obtemos a continuação analítica de $E_{1}^{M^{2}}(z ; a)$, a equação (146), pois as continuações analíticas da função gama, $\Gamma(z)$, e da função de Bessel modificada do segundo tipo, $K_{\alpha}(z)$, são bem conhecidas. Observe-se que a estrutura de pólos de $E_{1}^{M^{2}}(z ; a)$ fica determinada pela função $\Gamma(z-1 / 2)$. Deste modo, $E_{1}^{M^{2}}(z ; a)$ é uma função meromórfica com pólos simples situados em $z=\frac{1}{2},-\frac{1}{2},-\frac{3}{2}, \ldots$.

\section{Apêndice C: o propagador da partícula livre}

Comecemos com a representação de tempo próprio para o operador $\left(-\partial_{E}^{2}+m^{2}\right)^{-1}$ introduzida por
Schwinger [16]:

$$
\frac{1}{-\partial_{E}^{2}+m^{2}}=\int_{0}^{\infty} d s \exp \left[-\left(-\partial_{E}^{2}+m^{2}\right) s\right]
$$

onde $s$ é um parâmetro invariante com dimensões de comprimento ao quadrado. Outro resultado importante para os nossos propósitos é a equação de autovalores para o operador momentum linear ao quadrado no espaço euclidiano:

$$
\hat{P}^{2}|\omega, \mathbf{p}\rangle=p^{2}|\omega, \mathbf{p}\rangle,
$$

onde $p^{2}=\omega^{2}+\mathbf{p}^{2}$, e na representação de coordenadas $\hat{P}^{2} \rightarrow-\partial_{E}^{2}$. Portanto, para o propagador podemos escrever:

$$
\begin{gathered}
G_{0}\left(\tau-\tau^{\prime}, \mathbf{x}-\mathbf{x}^{\prime}\right)=\left\langle\tau, \mathbf{x}\left|\frac{1}{-\partial_{E}^{2}+m^{2}}\right| \tau^{\prime}, \mathbf{x}^{\prime}\right\rangle \\
=\int_{0}^{\infty} d s \exp \left(-m^{2} s\right)\left\langle\tau, \mathbf{x}\left|\exp \left[-\left(-\partial_{E}^{2}\right) s\right]\right| \tau^{\prime}, \mathbf{x}^{\prime}\right\rangle .
\end{gathered}
$$

Agora,

$$
\begin{gathered}
\left\langle\tau, \mathbf{x}\left|\exp \left[-\left(-\partial_{E}^{2}\right) s\right]\right| \tau^{\prime}, \mathbf{x}^{\prime}\right\rangle= \\
\int d \omega d^{3} \mathbf{p}\left\langle\tau, \mathbf{x}\left|\exp \left[-\left(-\partial_{E}^{2}\right) s\right]\right| \omega, \mathbf{p}\right\rangle\left\langle\omega, \mathbf{p} \mid \tau^{\prime}, \mathbf{x}^{\prime}\right\rangle \\
=\int d \omega d^{3} \mathbf{p} \exp \left[-p^{2} s\right]\langle\tau, \mathbf{x} \mid \omega, \mathbf{p}\rangle\left\langle\omega, \mathbf{p} \mid \tau^{\prime}, \mathbf{x}^{\prime}\right\rangle \\
=\int \frac{d \omega}{2 \pi} \frac{d^{3} \mathbf{p}}{(2 \pi)^{3}} \exp \left(-p^{2} s\right) \exp \left[i \omega\left(\tau-\tau^{\prime}\right)+\mathbf{p} \cdot\left(\mathbf{x}-\mathbf{x}^{\prime}\right)\right]
\end{gathered}
$$

onde fizemos uso da equação de autovalores para o operador $\hat{P},(\mathrm{C} 2)$, e suas autofunções na representação de coordenadas:

$$
\langle\tau, \mathbf{x} \mid \omega, \mathbf{p}\rangle=\frac{1}{(2 \pi)^{4}} \exp i(\omega \tau+\mathbf{p} \cdot \mathbf{x}) .
$$

Cada uma das quatro integrais no espaço euclidiano dos momentos que devemos calcular é da forma:

$$
\int_{-\infty}^{+\infty} d u \exp \left(a u^{2}+b u\right)=\sqrt{\frac{\pi}{-a}} \exp \left(-\frac{b^{2}}{4 a}\right)
$$

e o resultado da integração é:

$$
\left\langle\tau, \mathbf{x}\left|\exp \left[-\left(-\partial_{E}^{2}\right) s\right]\right| \tau^{\prime}, \mathbf{x} \prime\right\rangle=\left[\left(\frac{1}{4 \pi s}\right)^{\frac{1}{2}}\right]^{4} \exp \left(-\frac{\Delta^{2}}{4 s}\right),
$$

onde definimos $\Delta^{2}:=\left(\tau-\tau^{\prime}\right)^{2}+\left(\mathbf{x}-\mathbf{x}^{\prime}\right)^{2}$. Segue que:

$$
G_{0}\left(\tau-\tau^{\prime}, \mathbf{x}-\mathbf{x}^{\prime}\right)=\frac{1}{16 \pi^{2}} \int_{0}^{+\infty} \frac{d s}{s^{2}} \exp \left(-m^{2} s\right) \exp \left(-\frac{\Delta^{2}}{4 s}\right) .
$$

Agora fazemos uso da integral [5]:

$$
\int_{0}^{\infty} d u u^{\nu-1} \exp \left(-\gamma u-\frac{\beta}{u}\right)=2\left(\frac{\beta}{\gamma}\right)^{\frac{\nu}{2}} K_{\nu}(2 \sqrt{\beta \gamma}),
$$


onde $\Re \beta>0, \Re \gamma>0$ e $K_{\nu}(z)$ é uma função de Bessel modificada, obtendo:

$$
G_{0}\left(\tau-\tau^{\prime}, \mathbf{x}-\mathbf{x}^{\prime}\right)=\frac{m}{4 \pi^{2}|\Delta|} K_{1}(m|\Delta|)
$$

que é o propagador da partícula escalar livre em quatro dimensões euclidianas na representação de coordenadas.

\section{References}

[1] H.B.G. Casimir, Proc. K. Ned. Akad. Wet. 51, 793 (1948).

[2] M.J. Sparnaay, Physica 34 (1958) 751.

[3] S.K. Lamoreaux, Phys. Rev. Lett. 78, 5 (1997); erratum Phys. Rev. Lett. 81, 5475 (1988).

[4] U. Mohideen e A. Roy, Phys. Rev. Lett. 81, 4549 (1998).

[5] I.S. Gradshteyn and I. M. Ryzhik, Tables of Integrals, Series and Products, $5^{\text {th }}$ edition, (Academic Press, New York, 1994).

[6] A.P. French, Vibraciones y Ondas, (Editorial Reverté, Barcelona 1974).

[7] E. Elizalde e A. Romeo, Am. J. Phys. 59, 711 (1991).

[8] J.H. Cooke, Am. J. Phys. 66, 569 (1998).

[9] J.R. Ruggiero, A.H. Zimerman e A. Villani, Rev. Bras. Fís. 7, 663 (1977).

[10] A. Salam and J. Strathdee, Nuc. Phys B 90, 203 (1975); J.S. Dowker and R. Critchley, Phys. Rev. D 13, 3224 (1976); S.W. Hawking, Commun. Math. Phys. 55, 133 (1977); G. W. Gibbons, Phys. Lett. A 60, 385 (1977).

[11] E. Elizalde, S.D. Odintsov, A. Romeo, A.A. Bytsenko and S. Zerbini, Zeta Regularization Techniques with Applications, (World Scientific, Singapore, 1994).

[12] P.B. Gilkey, Invariance Theory, The Heat Equation and the Atiyah-Singer Theorem, $2^{\underline{a}}$ ed. (CTC Press, Boca Raton, 1995).

[13] M. Reuter e W. Dittrich, Eur. J. Phys. 6, 33 (1985).
[14] R.P. Feynman e A.R. Hibbs, Quantum Mechanics and Path Integrals (New York, McGraw-Hill, 1965).

[15] L.S. Brown e G.J. Maclay, Phys. Rev. 184, 1372 (1969).

[16] J. Schwinger, Phys. Rev. 82, 664 (1951).

[17] L.C. Albuquerque, C. Farina e L.G.A. Theodoro, Braz. J. Phys. 27, 488 (1997).

[18] S.K. Lamoreaux, Am. J. Phys. 10, 850 (1999).

[19] V.M. Mostepanenko e N.N. Trunov, Sov. Phys. Usp. 31, 965 (1988).

[20] V.M. Mostepanenko e N.N. Trunov, The Casimir Effect and its Applications, (Clarendon, Oxford, 1997).

[21] G. Plunien, B. Müller e W. Greiner, Phys. Rep. 134, 664 (1987).

[22] M. Bordag, U. Mohideen e V. M. Mostepanaenko; Phys. Rep. 353, 1 (2001).

[23] P.W. Milonni, The Quantum Vacuum: An Introduction to Quantum Electrodynamics, (Academic Press Inc., New York, 1994).

[24] T. Appelquist, A. Chodos e P.G.O. Freund (editores), Modern Kaluza-Klein Theories (Addison-Wesley Publishing Co., Reading, 1987).

[25] C. Itzkinson e J.B. Zuber, Quantum Field Theory (McGraw-Hill, New York, 1980).

[26] M.V. Cougo Pinto, C. Farina e A.C. Tort, Rev. Bras. Ens. Fís. 22, 122 (2000).

[27] P. Epstein, Math. Ann. 56, 615, (1903); 63, 205 (1907).

[28] S.D. Poisson, J. École Polytechnique XII, Cahier XIX, 420 (1823). 